\title{
Formation and Self- organization of Void Superlattices under Irradiation: a Phase Field Study
}

Yipeng Gao, Yongfeng Zhang, Daniel Schwen, Chao Jiang, Cheng Sun, Jian Gan

September 2018

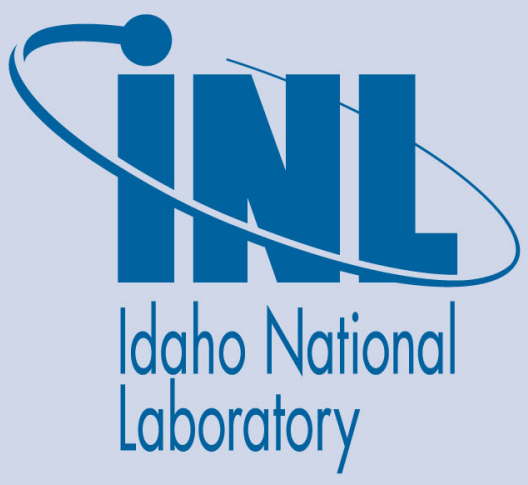

The INL is a U.S. Department of Energy National Laboratory operated by Battelle Energy Alliance 


\title{
Formation and Self-organization of Void Superlattices under Irradiation: a Phase Field Study
}

\author{
Yipeng Gao, Yongfeng Zhang, Daniel Schwen, Chao Jiang, Cheng Sun, Jian \\ Gan
}

September 2018

Idaho National Laboratory Idaho Falls, Idaho 83415

http://www.inl.gov

Prepared for the

U.S. Department of Energy

Under DOE Idaho Operations Office

Contract DE-AC07-05ID14517 


\title{
Formation and Self-organization of Void Superlattices under Irradiation: a Phase Field Study
}

\author{
Yipeng Gao, Yongfeng Zhang,* Daniel Schwen, Chao Jiang, Cheng Sun, and Jian Gan \\ Idaho National Laboratory (INL), Idaho Falls, ID 83415, USA
}

(Dated: April 19, 2018)

\begin{abstract}
Self-organized patterns, realized in non-equilibrium processes, have been widely observed in physics and chemistry. As a powerful tool to create far-from-equilibrium environments, irradiation produces a variety of types of defects, which can self-organize through physical interactions and chemical reactions. Such a process becomes complicated especially when both thermodynamics and kinetics play critical roles in pattern formation. In this paper, we investigate the formation and self-organization mechanism of void superlattices in metals and alloys under irradiation through phase field modeling and simulations. For the first time, three different formation mechanisms of void superlattices are clearly distinguished according to their thermodynamic origin and reaction kinetics. It is found that the characteristic length and symmetry of an emerging superlattice is determined by the interplay of the thermodynamic driving force and the kinetic anisotropy of the system. Through parametric study, the effects of kinetic coefficients, such as atomic mobility and irradiation dose rate, on the nucleation, growth, coarsening, coalescence, and ordering of voids are systematically investigated. The theoretical model developed in this work may provide guidelines for designing desired self-organized microstructures under irradiation.
\end{abstract}




\section{INTRODUCTION}

Self-organization phenomena occur in a number of different fields, including physics,

chemistry and biology, and they have attracted intense research interest. The spontaneous ordering processes during self-organization usually occur in open and complex systems that are at non-equilibrium states. Typical self-organization in physics includes phase transitions, such as ferroic (ferroelastic, ferroelectric, ferromagnetic) phase transitions, classical crystal growth, and Bose-Einstein condensation [1-6]. In literature, it is well known that the ordering and self-organization during phase transitions is directly associated with spontaneous symmetry breaking. For example, ferroelastic/ferroelectric/ferromagnetic domains are self-organized during phase transitions, and the formation of multi-domain structures is dictated by the minimization of long range interaction energies (elastic/electrical/magnetic energies). Note that both characteristic length and symmetry appear during such spontaneous ordering processes $[7,8]$. The characteristic length is determined by the interplay between the domain boundary energy and the long range interaction energy of a system, while the characteristic symmetry is determined by the broken symmetry. In other words, the self-organization during phase transitions is dominated by thermodynamics (or energetics). Similarly, a few modeling methods, such as the atomic density function theory and phase field crystal model, are developed to capture the atomic level characteristic length and symmetry (i.e., lattice parameter and crystal symmetry) [9-12]. In these methods, the free energy is formulated as a functional of atomic densities, which includes both shortrange and long-range thermodynamic interactions $[9,11-14]$. In contrast, the ordering and self-organization in reaction-diffusion systems in chemistry is usually dominated by kinetics (dynamics) [15-17]. In the Turing instability, for example, the ordering is dictated by the dynamic coupling between two (or more) components, which originates from chemical reactions. The competition between different temporal rates and spatial ranges of diffusion for different components is critical for the occurrence of dynamic instability. A characteristic length emerges in such a reaction-diffusion process, which is determined by the interplay of kinetic coefficients (e.g., diffusivity) and reaction terms. In addition, if the kinetic coefficients or reaction terms are associated with anisotropy (or symmetry breaking), characteristic symmetry is also expected during self-organization. Even though the above two different kinds of self-organizations may be distinguished in terms of thermodynamics and kinetics, real 
systems with both mechanisms involved could exit, especially in complex environments.

Irradiation is a powerful means to create complex environments and develop self-organized defect structures. On the one hand, as a continuous external stimulus maintains the system at states far from equilibrium, a large number of lattice defects of either vacancy or selfinterstitial-atom (SIA) type are generated during irradiation. These defects can be either isolated such as individual vacancies or SIAs, or agglomerate to form such as clusters and loops, etc. Along with these lattice defects, incorporation of impurities introduced by the implanting particles including ions and neutrons is possible. On the other hand, the internal interactions and reactions among those defects are activated, leading to the accumulation, annihilation, and organization of defects. Typical examples include dynamically-driven compositional patterning [18], the ordering of defect clusters and loops, and void and gas bubble superlattices in pure metals and alloys [19-23]. In general, self-organization under irradiation takes place as a result of the competition between the kinetics that drives the system towards equilibrium and the external stimuli that keep the system far from equilibrium. Such a competition has been well understood for the case of nanoscale compositional patterning [18]. However, a good understanding of void and bubble superlattices has yet to be established. In literature, several different theoretical approaches have been proposed to understand void superlattice formation. Parallel to phase transition and spinodal decomposition in solid and liquid solutions [24], thermodynamic descriptions of void formation have been suggested by Malen[25], Imada [26] and Veshchunov, et al [27], with however incomplete descriptions of defect production, reaction and annihilation. For instance, in Malen et al. [25], the void superlattice is regarded as a result of anisotropic elastic interactions among voids, without contributions from kinetic aspects. Although the elastic anisotropy could lead to ordering of voids, as indicated by 2D phase field simulations [28], the symmetry selection in 3D cases is not clear. In particular, the nucleation mechanism and development of superlattices in elastically isotropic tungsten cannot be well understood. Dynamic instability analysis based on the so-called rate theory is another popular approach that has been widely adopted in literature [29-32]. Rate theory captures the dynamic nature of the production, annihilation, and reactions of SIAs and vacancies as well as their agglomerates. However, a term analogous to Fickian diffusion is usually used for mass transport in this kind of analyses, and thus the thermodynamic origin of void formation is overlooked. In rate theory, the vacancy diffusion is driven by a concentration gradient. In contrast, the void formation in 
reality is through a diffusion against the concentration gradient, i.e., the diffusion is driven by the chemical potential gradient. As a consequence, the link between void formation and thermodynamic materials properties cannot be captured in rate theory. Theoretically, an analysis coupling both thermodynamics and kinetics is desired in the investigation of void lattice organization [33]. In particular, the pattern formation in self-organization could be related to the anisotropic diffusion of SIAs [33-39] or their clusters such as loops [40]. In fact, even without elastic anisotropy, 1D SIA diffusion is able to cause bubble superlattice formation, where a high ratio between the diffusivities of SIA and vacancy is suggested by 2D phase field simulations [38]. In addition, the role of 1D SIA diffusion in void superlattice formation has been further confirmed by atomic kinetic Monte Carlo (AKMC) simulations recently in both bcc and fcc crystals. It has been found that void superlattices can form as a result of spinodal decomposition of voids from the matrix, during which a characteristic length develops, with the simultaneous symmetry development dictated by the directions of SIA diffusion [33]. As the governing kinetics for the phase separations and defect reactions are different, i.e., the former by mass transport and the latter by the mutual recombination, the developments of characteristic length and symmetry of superlattices can occur at different stages. Prior to the present work, it has not been clearly demonstrated how these competing kinetics will affect the superlattice formation, and how the superlattice symmetry is determined at the continuum scale. Both of the above issues are the focus of this study.

In this work, we propose a model incorporating both the thermodynamic origin and the kinetic reactions during void superlattice formation under irradiation. By incorporating gradient thermodynamics and a Cahn-Hilliard type diffusion equation [41], a phase field model is developed to investigate the formation and evolution of void superlattices. A new scheme capturing the 1D SIA diffusion is proposed, without the calculations of the interstitial probability as in previous study [38]. The new approach is straightforward to implement in 2D and 3D with arbitrary anisotropy in SIA diffusion. As two examples, BCC and FCC types of void superlattices in 3D are obtained in phase field simulations. Consistent with previous studies, it is found that the characteristic length (related to the lattice parameter of the superlattice) is determined by an intrinsic thermodynamic instability influenced by the reaction kinetics, and the superlattice symmetry is dictated by the anisotropic diffusion of SIAs. Moreover, the competing kinetics for phase separation and defect reactions lead to three different formation mechanisms of void superlattices. By combining phase field simu- 
lations and theoretical analysis, a diagram capturing the selection of superlattice formation mechanisms is built with regards to irradiation conditions (e.g., dose rate) and reaction kinetics (e.g., mutual recombination).

\section{METHODOLOGY}

As mentioned above, both vacancy and SIA types of defects are generated in metals and alloys under irradiation. Depending on the size and configuration they take (point and cluster defects), these defects can be further divided into numerous types, and each type requires a rate equation to describe its evolution, similar to what has been done in previous instability analyses $[31,32,40]$. To simplify the theoretical analysis, we here identify the minimum number of essential factors that may be needed for void superlattice formation. To form voids, vacancy supersaturation in the matrix is necessary, which in turn needs defect production, transport, as well as the annihilation by recombination and sink absorption. A thermodynamic description of vacancies in the matrix and voids is needed as well. To simulate recombination, the evolution of SIAs needs to be considered, with its anisotropic diffusion governing the symmetry developed during phase separations. Following the mean field description, only the concentrations of vacancies and SIAs are considered in our theo-

retical model, which are the minimum factors required for void superlattice formation. The evolution of time- and spatially-dependent concentrations, $c_{v}$ and $c_{i}$ for vacancies and SIAs, respectively, are given by:

$$
\begin{array}{r}
\frac{\partial c_{v}}{\partial t}=\nabla \cdot M_{v} \nabla\left(\frac{\delta F}{\delta c_{v}}\right)-K c_{v} \sum_{i=1}^{n} c_{i}+P_{v}+\xi_{v} \\
\frac{\partial c_{i}}{\partial t}=\nabla \cdot D_{i} \nabla c_{i}-K c_{v} c_{i}+P_{i}+\xi_{i}, i=1,2, \ldots, n
\end{array}
$$

Here subscripts $v$ denote vacancy, and $i=1,2, \ldots, n$ denote the $i$ th type of SIAs. $P_{v}$ and $P_{i}$ are the production rates (related to the dose rate). $M_{v}$ and $D_{i}$ denote the atomic mobility of vacancies and the diffusivity of SIAs, respectively. $F$ is the total free energy of the system. $K$ is the reaction rate for recombination. In Eq. 1, we further ignore sink absorption in its isotropic form, which could affect vacancy accumulation rate but not significantly the competition between phase separation and mutual recombination. The reaction rate 
for recombination is reduced to a constant $K$ to better elucidate the competing kinetics. In reality it is given by $K=\frac{4 \pi R_{i v}\left(D_{i}+D_{v}\right)}{\Omega}$, where $R_{i v}$ is the recombination radius, $D_{v}$ is the vacancy diffusivity, and $\Omega$ is the atomic volume. $\xi_{v}$ and $\xi_{i}$ are Langevin noise terms simulating fluctuations in vacancy and SIA concentrations, which meet the fluctuationdissipation theorem.

Our model couples the rate theory for production and reaction kinetics [42], and the Cahn-Hillard approach for the phase separation description of void formation [24, 28]. In phase field model, a void is described as a new "phase", the formation of which is the result of vacancy diffusion and accumulation [38, 43-54]. In addition, $n$ types of SIAs are introduced in our new approach, each of them diffusing anisotropically along a particular crystallographic direction, which could be related to the symmetry of the host matrix. For example, in a bcc host matrix, if the SIAs diffuse in 1D along $\langle 111\rangle$, there are four types of SIAs $(n=4)$, which diffuse along [111], [11 1$],[1 \overline{1} 1],[\overline{1} 11]$, respectively. In an fcc host matrix, if the SIAs diffuse in 1D along $\langle 110\rangle$, there are six types of SIAs $(n=6)$, which diffuse along [110], [011], [101], [1]10], [01]], [101], respectively. Note $P_{v}=\sum_{i=1}^{n} P_{i}=P$, i.e., irradiation produces the same amount of vacancies and SIAs due to mass conservation. And the production rate for each type of SIAs should be $P / n$, since all types of SIAs have the equal probability of being produced by irradiation.

The total free energy $F$ can take different forms depending on the nature of the diffusion process. In this case, the void formation due to vacancy accumulation can be described in a way similar to a phase separation. In general, the total free energy in an inhomogeneous system is formulated based on gradient thermodynamics [41],

$$
F=\int_{V}\left(f+\frac{1}{2} \kappa\left|\nabla c_{v}\right|^{2}\right) d V
$$

$f$ is the local free energy density simplified to a double-well function in a dimensionless form as follows, and $\kappa$ is the coefficient of gradient energy, which is associated with the interfacial energy (interface between void and matrix).

$$
f^{*}\left(c_{v}\right)=c_{v}^{2}\left(1-c_{v}\right)^{2}
$$

Note that Eq. 4 is a generic form for phase separations, which can capture both nucleation/growth process (with thermal noise combined) and spinodal decomposition. For ex- 
ample, when the initial concentration is within the spinodal region $\left(0.2113<c_{v}<0.7887\right)$, spinodal decomposition occurs without thermodynamic barrier. Otherwise, nucleation and growth are expected as those in conventional first-order transitions. The above simplified equation of free energy density is chosen because: (1) an analytic prediction of the characteristic length of void superlattices can be obtained; (2) it is a representative formulation that can be solved efficiently in phase field modeling and simulations. In order to analyze the void superlattices observed in experiments, more accurate energetic descriptions should be employed, as will be shown in our discussions.

A phase field model to investigate the void superlattice formation is developed based on the open source MOOSE finite element framework $[55,56]$. In MOOSE, the phase field kinetic equations (e.g., Cahn-Hilliard equation) are implemented in a general form which is separated from the thermodynamic information. In addition, the spatial discretization is finite-element-based, which can accommodate various kinds of geometries and boundary conditions. Implicit time integration is utilized with adaptive time stepping, employing the preconditioned Jacobian-free Newton Krylov method and utilizing capabilities provided by the libMesh and PETSc libraries [57, 58].

Without loss of generality, all simulations are performed using dimensionless parameters. The kinetic equations dominating the evolution of dimensionless parameters are the same as Eq. 1, with $M_{v}, D_{i}, f, K$ and $P$ substituted by their dimensionless counterparts (i.e., $M_{v}^{*}, D_{i}^{*}, f^{*}, K^{*}$ and $\left.P^{*}\right)$. In the following sections, the analytical derivation is based on real material parameters so that the predicted superlattice parameters can be directly compared with experimental observations. In phase field models, the relation between real parameters and dimensionless parameters determines the length and time scales of the simulations [59], provided the thermodynamic and kinetic data are available. The gradient coefficient $\kappa(\mathrm{J} / \mathrm{m})$ can be determined from the interfacial energy [41, 59], and the unit dimensionless length corresponds to a real length of

$$
l_{0}=\sqrt{\frac{\Delta f^{*} \cdot \kappa}{\Delta f \cdot \kappa^{*}}}
$$

where superscript $*$ indicates the dimensionless parameters. $\Delta f$ is energy barrier between void and matrix phases, in the unit of $J / \mathrm{m}^{3}$. Thus the unit dimensionless time corresponds to a real time of 


$$
t_{0}=\frac{M_{v}^{*} \cdot \Delta f^{*}}{M_{v} \cdot \Delta f} l_{0}^{2}
$$

Then the dimensionless production rate and recombination rate can be determined through

$$
\frac{P^{*}}{P}=\frac{K^{*}}{K}=t_{0}
$$

The dimensionless production rate $P^{*}$ and recombination rate $K^{*}$ are determined by given real defect production rate $P$, recombination rate $K$, and $t_{0} . P$ is in the unit of $d p a / s$ (when $t_{0}$ is in $s$ ), with dpa being displacement per atom and carrying the same unit of $c_{v}$. $P$ represents the effective dose rate in reality.

\section{RESULTS}

In our model a set of concentration order parameters $\left\{c_{v}, c_{1}, c_{2}, \ldots, c_{n}\right\}$ is employed to describe the defect evolution in materials. $c_{v}=1$ indicates the formation of a void. A two-dimensional (2D) computational system with 10000 quad elements is constructed, with a homogeneous configuration $\left\{c_{v}, c_{1}, c_{2}, \ldots, c_{n}\right\}=0$ as the initial condition. Langevin noise terms are employed to initiate the phase separation, the dimensionless form of which is represented by a Gaussian function, with an expected value of zero and a variance of $10^{-5}$ in the simulations. All the dimensionless parameters used in the simulations are listed in TABLE I. As will be shown in the discussion, the formation of void superlattices occurs in a specific parameter space for vacancy mobility, production and recombination rates. Outside that parameter space, superlattice cannot form.

\section{Formation of square void superlattices}

In 2D the SIA diffusion directions are the [10] and [01] directions (e.g., in a square lattice host matrix), with diffusivity tensors in the following forms: 


$$
\begin{aligned}
& D_{1}^{*}=\left(\begin{array}{cc}
10 & 0 \\
0 & 0.01
\end{array}\right) \\
& D_{2}^{*}=\left(\begin{array}{cc}
0.01 & 0 \\
0 & 10
\end{array}\right)
\end{aligned}
$$

First we consider a case with a relatively high production rate $\left(P^{*}=0.016\right)$ and $K^{*}=0.16$ as the dimensionless recombination rate of vacancies and interstitials. The evolution of the vacancy concentration field is shown in FIG.1. In FIG. 1(a) and (b) fluctuations of the vacancy concentration can be observed before void formation occurs, which is caused by the thermodynamic instability described in Eq. 4. As the vacancy concentration increases to a certain extent (i.e., approaching the spinodal region), the homogeneous vacancy distribution becomes unstable with respect to infinitesimal fluctuations and concentration waves can develop. When the amplitudes of the vacancy concentration waves are large enough, voids form at the sites with high vacancy concentrations (FIG. 1(c)). We observe that characteristic length and symmetry of a square superlattice are developed at the beginning of vacancy modulation (FIG. 1(a)), which determines the initial distribution of voids (FIG. $1(\mathrm{c})$ ). Coalescence of voids can also be observed during the evolution, e.g., the two rows of voids on the top coalesce to one row (FIG. 1(c)-(e)). Finally, a stable void superlattice forms (FIG. 1(f)), without further coarsening and coalescence because of the balance of production and recombination.

To further understand the formation mechanisms of voids and void superlattices, we plot the concentration fields of the two types of SIAs in FIG. 2. The concentration fields of $c_{1}$ at dimensionless time $t^{*}=836,1167,2000$ are shown in FIG. 2(a)-(c), while those of $c_{2}$ are shown in FIG. 2(d)-(f), which corresponds to the microstructures in FIG. 1(a), (d) and (f). The SIA concentration field $c_{1}$ exhibits a horizontal layer structure with vertical modulations (FIG. 2(a)-(c)). Similarly, the $c_{2}$ field has a vertical layer structure with horizontal modulations (FIG. 2(d)-(f)). The modulation direction is associated with the 1D diffusion direction for each type of SIA. For example, the 1D diffusion direction of $c_{1}$ is [10], resulting in a layer structure along the [10] direction, while the modulation direction is normal to [10]. The coupling between vacancies and SIAs can also be observed. Considering the superposition of FIG. 2(c) and (f), a 2D modulated structure can be expected. Such a modulated 
structure should be directly related to the void superlattice in FIG. 1(f), i.e., a void forms where the SIA concentration is zero, which is due to the recombination of vacancies and SIAs. The modulations of SIAs appear to coincide with vacancy modulations (comparing FIG. 2(a) and (d) with FIG. 1(a)). As we can see in the final microstructure (FIG. 2(c) and (f), FIG. 1(f)), the SIA concentration in voids is zero due to the recombination of vacancies and SIAs. This suppresses SIA transport across voids in our model, without explicitly building this into the kinetic equations.

Results of another simulation with relatively low production and recombination rates $\left(P^{*}=0.006\right.$ and $\left.K^{*}=0.06\right)$ are shown in FIG. 3. Because of less ordering in the initially formed void superlattice in FIG. 3(a) (compared with FIG. 1(c)), significant coarsening and coalescence can be observed in FIG. 3(a)-(e). The evolution stops when a perfect superlattice structure forms (FIG. 3(f)). Compared with FIG. 1(f), both the void size and the lattice parameter in FIG. 3 are larger because of the coarsening and coalescence of voids.

In both of the above cases, the production rate is sufficiently high so that the vacancy concentration can go into the spinodal region $(\sqrt{P / K}>0.2113)$. Therefore, phase decomposition can occur before or along with the ordering of the voids. However, this condition is not necessarily satisfied especially when the production rate is low and the recombination rate is high. Because of the balance of the production and recombination of vacancies and SIAs, the steady state homogeneous vacancy concentration could be outside the spinodal region. In such a case, we cannot expect spinodal decomposition any more. Instead, a nucleation and growth mechanism could occur for voids to form, as shown in FIG. 4. In this simulation, the production and recombination rates are chosen as $P=0.007, K=0.16$. A single void nucleates first in FIG. 4(a). As the first void grows larger, another void nucleate at a position being aligned with the existing one (FIG. 4(b)), due to a 1D SIA diffusion shadow. With more and more voids nucleating in alignment, the system finally evolves to a defect-rich superlattice (FIG. 4(c)-(f)). In this case, ordering of voids takes place first.

\section{Formation of hexagonal void superlattice}

To explore the effects of host matrix symmetry and the directions of SIA diffusion we

considered the case of a hexagonal lattice. Here, SIAs diffuse along [10], [-1, $\sqrt{3}]$ and $[-1,-\sqrt{3}]$ directions (e.g., in a hexagonal lattice host matrix) with diffusivity tensors in the 
following forms:

$$
\begin{array}{r}
D_{1}^{*}=\left(\begin{array}{cc}
10 & 0 \\
0 & 0.01
\end{array}\right) \\
D_{2}^{*}=R^{T} D_{1} R \\
D_{3}^{*}=\left(R^{2}\right)^{T} D_{1} R^{2},
\end{array}
$$

where $R$ is the matrix for a $120^{\circ}$ rotation and superscript $T$ means the matrix transpose.

The simulation results are shown in FIG. 5. As a result of a change in SIA 1D diffusion directions, a hexagonal superlattice forms, which is different from the square superlattice previously obtained. We observe that the initial void distribution is far from a perfect superlattice (FIG. 5). Through coarsening and coalescence it evolves to a perfect hexagonal superlattice (FIG. 5(a)-(f)). During this process, not only individual voids but also entire void rows could disappear (circled in FIG. 5(c) and (e)).

\section{Formation of BCC and FCC void superlattices}

The effects of SIA diffusion directions on the superlattice symmetry are also investigated in 3D. In Fig. 6 (a), four types of SIAs are considered with diffusion direction $\langle 111\rangle$, leading to the formation of a BCC superlattice. In Fig. 6 (b), six types of SIAs are considered with diffusion direction $\langle 110\rangle$, leading to the formation of an FCC superlattice. The formation of BCC and FCC superlattice demonstrate that our new approach is sufficiently general to capture various kinds of anisotropic SIA diffusions in both 2D and 3D. A detailed discussion on the symmetry selection of void superlattices will be presented in the next section.

\section{DISCUSSION}

From a theoretical point of view, a void superlattice features certain lattice parameters and lattice symmetries, which vary with materials and irradiation conditions (e.g., temperature and dose rate) [23]. To understand the formation mechanism of void superlattices, we further investigate the effects of materials parameters and irradiation conditions on the characteristic length and symmetry of void superlattices. The results presented in the previous section clearly indicate three different kinds of formation mechanisms: (I) high density 
of voids form via spinodal decomposition with the concurrent development of characteristic length and symmetry; (II) high density of voids form via spinodal decomposition without ordering, which develops during coarsening and coalescence; (III) isolated voids form through nucleation with spatial alignment, and superlattices form by further nucleation and growth of voids with the same alignment. These different mechanisms are caused by the competition between the kinetics for phase separations which can occur by either spinodal decomposition or void nucleation and growth, and the kinetics for ordering which is induced by the recombinations. As will be discussed below, the competition affects both superlattice formation mechanisms and superlattice parameters. For the sake of computational efficiency and consistency, square superlattices in 2D are considered in the following.

\section{Characteristic length of the void superlattice}

For different formation mechanisms, the governing kinetics for the characteristic length (thus the superlattice parameter) development varies. For mechanism (I), a characteristic length develops during spinodal decomposition and is stabilized by the concurrent development of ordering. In this case a theoretical analysis can be done to predict the superlattice parameter, as has been done in our previous work [33]. The derivation is provided here as well for completeness as the kinetic equation is different here.

Using $Q=K \sum_{i=1}^{n} c_{i}$, Eq. 1 can be reduced to

$$
\frac{\partial c_{v}}{\partial t}=\nabla \cdot M_{v} \nabla \frac{\delta F}{\delta c_{v}}-Q c_{v}+P
$$

In the above form, Eq. 13 is a generalized diffusion equation (Cahn-Hilliard type) with a source term $(P)$ and a sink term $\left(Q c_{v}\right)$. Only the first term involves the thermodynamic factor, which is scaled by the kinetic coefficient of mobility, capturing the spatial migration

of vacancies. Note that the reaction term could be nonlinear since $c_{i}$ in $Q$ are coupled with $c_{v}$. For simplicity, $M_{v}$ and $Q$ are taken as constants, and Eq. 13 becomes a linear partial differential equation of $c_{v}$, which can be analyzed in reciprocal space through Fourier transform,

$$
\frac{\partial \tilde{c}_{v}}{\partial t}=\left[-M_{v} f^{\prime \prime} k^{2}-M_{v} \kappa k^{4}-Q\right] \tilde{c}_{v}+P \delta(k)=R(k) \tilde{c}_{v}+P \delta(k)
$$


where $\tilde{c}_{v}$ is the Fourier transform of $c_{v}$, and $k$ is the magnitude of the wave vector $(k=|\boldsymbol{k}|)$ in reciprocal space. $f^{\prime \prime}$ is the second-order derivative of the free energy density function. Note that the last term in Eq. 14 is nontrivial only when $k=0$, i.e., the uniform change of concentration, which does not contribute directly to the instability caused by the phase separation. For $k \neq 0$,

$$
R(k)=-M_{v} f^{\prime \prime} k^{2}-M_{v} \kappa k^{4}-Q
$$

At a given $k$, if $R(k)>0$, the system will lose stability with respect to infinitesimal concentration fluctuation for the wave with the wave vector magnitude $k$, i.e., such a wave will develop. For the first developed wave with $k_{c}, R(k)$ should reach the maximum at $k_{c}$. By solving the critical conditions for $k_{c}$, the critical wavelength can be determined [33] (see Appendix A),

$$
\lambda_{c}=\frac{2 \pi}{k_{c}}=2 \pi\left(\frac{\kappa M_{v}}{Q}\right)^{1 / 4}
$$

The critical concentration wavelength determines the lattice parameter of the void superlattice initially formed under irradiation, which is related to the 1D SIA diffusion direction. For example, $\lambda_{c}=a_{s q}$ in a 2D square lattice, $\lambda_{c}=(\sqrt{3} / 2) a_{h e x}$ in a 2D hexagonal lattice, $\lambda_{c}=(\sqrt{2} / 2) a_{B C C}$ in a 3D BCC lattice, and $\lambda_{c}=(\sqrt{3} / 3) a_{F C C}$ in a 3D FCC lattice.

To validate our theoretical predictions, we perform a series of phase field simulations with different recombination rates and vacancy mobilities. The parameters are chosen appropriately for the mechanism (I) to be effective. The resulting superlattices are shown in FIG. 7. The comparison between simulated superlattice parameters and analytical predictions using Eq. 16 are presented in TABLE II. In the theoretical calculations $Q=K \sqrt{P / K}$ (corresponds to the steady state vacancy and SIA concentrations before modulation) and $P^{*}=0.016$ are used for all the four cases.

It can be found that the theoretical predictions are in good agreement with the phase field simulation results both qualitatively and quantitatively. The simulation results are slightly above the theoretical predictions. One possible reason is the periodic boundary condition used in the simulations. Note that the number of void rows has to be an integer, so that the lattice parameter can only be discrete (e.g., ..., 16, 13.33, 11.44, 10, 8.89, 8, 7.27, 6.67, ...) in the simulations. If the predicted critical wavelength by Eq.16 is not exactly the above value, 
coarsening and coalescence may increase the wavelength to the nearest lattice parameter compatible with the periodic boundary condition.

To compare the results to real materials, Eq. 16 is also utilized to estimate the lattice parameters of void superlattices in molybdenum at $580^{\circ} \mathrm{C}$ and $900^{\circ} \mathrm{C}$ [33]. The free energy density $f$ as a function of temperature can be approximated by a regular solution model [60]. $\kappa=3.92 \times 10^{-10} \mathrm{~J} \cdot \mathrm{m}^{-1}$ can be determined, which is associated with the interfacial energy $[59,61]$. At $580^{\circ} \mathrm{C}, M_{v}=1.33 \times 10^{-25} m^{5} \cdot J^{-1} \cdot s^{-1}$ is determined from the vacancy diffusivity $[60,62] . Q$ is estimated to be $0.374 \mathrm{~s}^{-1}$, so that the critical wavelength is $21.6 \mathrm{~nm}$, which corresponds to a lattice parameter in $\mathrm{BCC}$ about $30.5 \mathrm{~nm}[32,33,42]$. At $900^{\circ} \mathrm{C}$, $M_{v}=2.10 \times 10^{-23} \mathrm{~m}^{5} \cdot J^{-1} \cdot \mathrm{s}^{-1}$. $Q$ is estimated to be $10.2 \mathrm{~s}^{-1}[33,60,63]$, so that the critical wavelength is $33.5 \mathrm{~nm}$, which corresponds to a lattice parameter about $47.4 \mathrm{~nm}$ in BCC. In experimental observations, the lattice parameters of void superlattices in molybdenum are determined as $27 \mathrm{~nm}$ and $49 \mathrm{~nm}$, at $580^{\circ} \mathrm{C}$ and $900^{\circ} \mathrm{C}$, respectively [23, 64]. Detailed calculations are presented in Appendix B.

The analytical solution predicts the formation of a void superlattice following Mechanism (I) with concurrent spinodal decomposition and ordering very well. For mechanism (II), Eq.16 is still suitable to predict the wave length selection during spinodal decomposition. As the void superlattice is stabilized during subsequent coarsening and coalescence, the resulting superlattice parameter will be larger than that given by Eq.16. In this case, $\lambda_{c}$ is governed by coarsening kinetics. As for mechanism (III), because the system may not enter the spinodal region, the critical wavelength is not applicable anymore. Instead, $\lambda_{c}$ is determined by void nucleation and growth kinetics.

\section{Selection of the formation mechanisms}

Theoretically, when $R(k)<0$ for all $k$, Mechanisms (I) or (II) will not be expected. With the simplified model of Eq. 1, the maximum value of $c_{v}$ is $\sqrt{P / K}$, which corresponds to a steady state homogeneous vacancy concentration (due to dynamic balance between vacancy production and recombination). In order to develop vacancy modulations as well as a superlattice through spinodal decomposition, the maximum $c_{v}$ should be inside the spinodal region. In addition, the thermodynamic driving force of the spinodal decomposition should be large enough to overcome the barrier from recombination (See Appendix A). 


$$
\max \left\{-f^{\prime \prime}\left(c_{v}\right), c \in[0, \sqrt{P / K}]\right\} \geq \sqrt{\frac{4 \kappa \sqrt{P K}}{M_{v}}}
$$

When this condition is satisfied, the competition between mechanisms (I) and (II) can be elucidated by examining the kinetics for the phase decomposition, controlled by the production rate $P$ and the vacancy mobility $M_{v}$, and that for recombination, controlled by the recombination rate $K$. In particular, when the maximum value of $f^{\prime \prime}$ is reached at $\sqrt{P / K}$, and also the equality in Eq. 17 is reached, the above inequality becomes

$$
-f^{\prime \prime}(\sqrt{P / K})=\sqrt{\frac{4 \kappa \sqrt{P K}}{M_{v}}}
$$

The above equation describes the ideal condition for phase decomposition and void ordering to take place concurrently. In such a condition, perfect superlattices are expected directly from spinodal decomposition without coarsening. The superlattice parameters are predictable using Eq. 16. In fact, the simulation results in FIG. 7 are obtained in this condition. Note that a critical value of $M_{v}$ could be determined by Eq. 18 for given $P, K$ and $f$. If $M_{v}$ is smaller than the critical value, there will not be vacancy concentration modulation since Eq. 17 will not be satisfied. Increasing in $M_{v}$ will accelerate phase separation kinetics (relative to recombination), leading to imperfect superlattices from initial phase separation.

To further elucidate the effects of vacancy mobility, production, and recombination rates on the selection of superlattice formation mechanism, parametric studies are performed with varying $P$ and $M_{v}$. In FIG. 8, the vacancy mobility is increased $\left(M_{v}^{*}=3\right.$, compared to $M_{v}^{*}=0.3$ in FIG. 1 ). Because of a larger mobility, vacancy concentration modulation develops faster, with less influence from the recombination between vacancies and SIAs. In other words, vacancy mobility dominates the kinetic competition between $M_{v}$ and $K$. Since the symmetry of a void superlattice is mainly contributed from the recombination, no clear symmetry is developed at the initial void formation (FIG. 8(b)). The Fourier transform patterns are shown as insets for corresponding microstructures. The origin $(\boldsymbol{k}=(0,0))$ in reciprocal space is not considered, since it does not include any modulation information. It can be found that a ring-like pattern forms at the beginning (FIG. 8(a)), which gradually evolves to individual spots in FIG. 8(b)-(f). Such results suggest that only a characteristic wavelength (related to the radius of ring pattern) is developed at the beginning, while a characteristic symmetry is developed during the subsequent coarsening and coalescence. In 
contrast, a superlattice close to perfect is developed during the initial formation in FIG. 1(c), with all the voids at almost the same size (and a uniform inter-void spacing). As a result, only minor adjustments are observed in the subsequent evolution. Note that when a perfect superlattice is formed, coarsening and coalescence are suppressed as the system has entered a thermodynamically metastable state.

In Mechanism (I) and (II), the production rate is sufficiently high so that the vacancy concentration can go into the spinodal region $(\sqrt{P / K}>0.2113)$. However, this condition is not necessarily satisfied especially when the production rate is low and the recombination rate is high. Because of the balance of production and recombination, the steady state homogeneous vacancy concentration could be outside the spinodal region. In such a case, we cannot expect spinodal decomposition any more. Instead, a nucleation and growth mechanism could occurs, and the formation of a superlattice depends on the interplay between nucleation rate and growth kinetics, through Mechanism (III).

A typical superlattice formation process through void nucleation and growth is shown in FIG. 9 and FIG. 10. With an existing void, a new void tends to nucleate at a position aligned (either horizontally or vertically) with an existing one (FIG. 9(a)), which is due to the 1D SIA diffusion (FIG. 10). The alignment of voids finally evolves into a superlattice (FIG. 9(b)-(f)). From the Fourier transform patterns, some symmetry is developed first ((FIG. 9(b) and (c))), and the characteristic length appears during subsequent nucleation and growth. The characteristic length finally developed is dictated by the interplay between nucleation rate and vacancy mobility. Note the difference between FIG. 9 and FIG. 4. In FIG. 4, we can see that the first void grows larger when the second one nucleates, because of a higher vacancy mobility $\left(M_{v}^{*}=1\right)$. As a result, both the void size and the inter-void spacing becomes larger, which leads to a larger lattice parameter of the final void superlattice (FIG. 4).

The selection of the formation mechanism is dictated by the interplay among vacancy mobility, the recombination rate, and the production rate, which correspond to the three terms in Eq. 1, respectively. The competition between $P$ and $K$ determines if the system enters the spinodal region. The competition between $M_{v}$ and $K$ determines if phase separation is accomplished earlier than void ordering, or otherwise. Accordingly, diagrams describing the selection of void superlattice mechanisms with respect to $P$ and $K$ and $M_{v}$ can be constructed. An example of such diagram with respect to $P$ and $K$ at a given $M_{v}^{*}$ 
is shown in FIG. 11. Simulation results of void superlattice formation through different mechanisms are represented by dots with different colors. The void superlattices formed through mechanism (I) are colored by red, (II) blue and (III) green. The solution of Eq. 18 is described by the red line, representing the ideal condition (on $P$ and $K$ ) for Mechanism (I), from which a small deviation will not change the dominant mechanism. With sufficient deviation, the formation mechanism changes to (II) when $P$ is high and $K$ is low, and to (III) when $P$ is low and $K$ is high. The boundaries among those three regions are sketched by using black dashed lines. Note that no boundaries are suggested on the left of region (II) and bottom of region (III). With very low production or low recombination, superlattice formation may take very long time or even not occur due to low vacancy accumulation or insufficient ordering. Even though the effect of vacancy mobility $M_{v}$ is not explicitly shown in FIG. 11, it can be deduced from the diagram as well. Since there are three linear terms in Eq. 1, increasing $M_{v}$ should be equivalent to decreasing $P$ and $K$. Note that all the lines (the red line and the black dash lines) are non linear. For example, $\left(P^{*}=0.016, K^{*}=0.16\right)$ is in region (I) while $\left(P^{*}=0.006, K^{*}=0.06\right)$ is in region (II). As a result, an increase of $M_{v}$ could change the formation mechanism from (I) to (II) (comparing FIG. 8 and FIG. 1). In addition to such a "shift" effect, an increase of $M_{v}$ could also change the growth rate in Mechanism (III) (comparing FIG. 9 and FIG. 4).

In reality, the three different regions in FIG. 11 represent the formation mechanisms under different irradiation conditions. In experiments, temperature and dose rate are the parameters that can be controlled. The major effect of temperature is the change of vacancy mobility, i.e., mobility is high at high temperature. The effect on recombination rate is less significant since recombination is dominated by SIA diffusion, which is usually associated with very a low barrier and thus weak temperature dependence. However, an increase in temperature may change the anisotropy of SIA diffusion, which transitions from 1D to 3D. According to Eq. 18 and FIG. 11, a balance exists between $P, M_{v}$ and $K$ for the ideal condition of a void superlattice formation, as suggested by Eq. 18 and the red line in FIG. 11. Near this condition mechanism (I) dominates. An increase in dose rate and temperature will shift the formation mechanism to (II). Without the available data on the sink absorption and thermodynamics of SIAs, the SIA concentrations could be over estimated in the current simulations, so that recombination as well as void ordering are enhanced. Therefore, the condition for mechanism (III) may be difficult, if not impossible, to realize in experiments. 
From theoretical point of view, Eq. 18 (red line in FIG. 11) can be considered as the ideal condition for void superlattice formation. The formation mechanism changes (from I to II or III) if there is a small deviation from the ideal condition. A large deviation will result in the absence of void superlattices. The above three different mechanisms are also suggested by experimental observations. An imperfect void superlattice is observed in $\mathrm{Nb}$, in a high dose rate $\sim 2 \times 10^{-3} \mathrm{dpa} / \mathrm{s}$ and medium temperature $T / T_{m} \sim 0.37$ [65], which is consistent with the microstructure generated through Mechanism II. Void alignments rather than a superlattice are observed in $\mathrm{Cu}-\mathrm{Ni}$, in a low dose rate $\sim 3.5 \times 10^{-7} \mathrm{dpa} / \mathrm{s}$ and high temperature $T / T_{m} \sim 0.51$ [66], which indicates the Mechanism III. To obtain a perfect superlattice through Mechanism I, a high dose rate and low temperature are required, which is consistent with the observations in U-7Mo system, in a nuclear fuel irradiated in research reactors at $T / T_{m} \sim 0.28[67]$.

\section{Characteristic symmetry of the void superlattice}

From the above simulation results, it is clear that the symmetry of a void superlattice is directly related to the 1D SIA diffusion directions. Parallel to the symmetry breaking concept in phase transitions, the self-organization of voids originates from the symmetry breaking of diffusivity from isotropic to anisotropic. And the symmetry of a void superlattice should be determined by the broken symmetry. The broken symmetry can be described by $1 \mathrm{D}$ SIA diffusion directions in real space, which corresponds to $k$-planes in reciprocal space. Simulation results of void superlattices as well as their Fourier transform patterns for different kinds of anisotropic diffusivities are shown in FIG. 12.

In FIG. 12(a), the diffusivity tensors are those described by Eq. 8 and 9 . Note that diffusivity is a second-rank tensor, which has central symmetry, so that the diffusivities of different types of SIAs in this case are related by 4-fold rotational symmetry (double the number of SIA types). In real space, the 1D SIA diffusion directions are [10] and [01], which correspond to $k$-planes of $(10)$ and (01) in reciprocal space $(k$-points, $k$-planes and $k$-lattice refer to the points, planes and lattice in reciprocal space). Considering a set of parallel (10) $k$-planes and a set of parallel (01) $k$-planes, their intersection $k$-points construct a square $k$-lattice. The inverse Fourier transform of such a square $k$-lattice is a square lattice in real space, which is the void superlattice in FIG. 12(a) with 4-fold rotational symmetry. 
When the diffusivity tensors are described by Eq. 10-12, the 1D SIA diffusion directions are $[1,0],[-1, \sqrt{3}]$ and $[-1,-\sqrt{3}]$. Similar to the above analysis, a hexagonal $k$-lattice can be constructed by three sets of $k$-planes, which correspond to a hexagonal void superlattice in real space with 6-fold rotational symmetry (in FIG. 12(b)).

The above analysis can be easily generalized to 3D. For example, if the 1D SIA diffusion direction is $\langle 111\rangle,(1,1,0)$ type of $k$-points are determined by four $\{111\} k$-planes, which leads to an FCC $k$-lattice, corresponding to a BCC lattice in real space. In fact, it has been observed in experiments that a 1D SIA diffusion along [111] does not cause the order of voids along [111] [68], but ordering of $\{110\}$ planes. As it is suggested by our simulation results (FIG. 2), a 1D SIA diffusion along [111] leads to the modulation of all the directions normal to [111], corresponding to the (111) $k$-plane in reciprocal space. Similarly, if the 1D SIA diffusion direction is $\langle 110\rangle,(1,1,1)$ types of $k$-points are determined by six $\{110\} k$-planes, which leads to a BCC $k$-lattice, corresponding to a FCC lattice in real space. Also based on the analysis in reciprocal space, the characteristic length of a superlattice is directly related to the inter-plane spacing in real space, since it is dictated by the $k$-lattice.

Note that the characteristic symmetry of a void superlattice is related to the 1D SIA diffusion directions, regardless of the formation mechanisms of (I) (II) and (III).

\section{Limitations}

Several major simplifications warrant a discussion, e.g., SIA thermodynamics and sink absorption are not explicitly considered. Such a treatment may lead to an over estimate of SIA concentrations at the steady state. In our simulations, the steady state concentration of vacancies and SIAs are at the same order of magnitude. In reality, the value of vacancy concentration should be orders of magnitude larger than the SIA concentration, caused by the different sink absorption rates of vacancy and SIA. Effectively, high SIA concentrations delays vacancy accumulation and enhance void ordering since here SIA diffusion is purely anisotropic. We do not consider the transition of SIAs between different diffusion directions, e.g. the rotation of crowdions, which would effectively reduce the degree of anisotropy in the diffusion. Also, the clustering of SIAs and formation of SIA loops are not considered as well due to the lack of thermodynamic description of SIAs. As discussed in details in literature $[38,39]$, the ratio between the diffusivities of SIA and vacancy, $D_{S I A} / D_{v}$, plays a 
role on the ordering of voids. In our simulations we focus on the the selection mechanism of void superlattice formation, and we assume $D_{S I A}$ is large enough so that it will not be the limiting factor for the superlattice formation. This simplification can be refined if the diffusivity data for real material system is available. In addition, a simplified free energy density formulation is adopted for vacancies in our model, which is convenient for both analytical derivations and numerical simulations. Such a free energy could be substituted by more accurate physical formulations (e.g., regular solution model) for systems with available thermodynamic and kinetic data. These assumptions are used to simply the problem to include only several essential factors for void superlattice formation and to elucidate the nature of their competition. They may limit the direct application of the current results in real material systems. However, we argue that our major findings, such as the different superlattice formation mechanisms resulting from the competing kinetics and the lattice symmetry selection as a result of anisotropic SIA diffusion, will still hold in case without these assumptions.

In literature, a number of analytical methods $[69,70]$ have been developed to predict the characteristic length and symmetry of an ordered and self-organized system. In those methods, the characteristic length and symmetry are determined by thermodynamic equilibrium in closed systems. However, this work considers an open system under irradiation in this work, and continuous external stimuli keep the system far from equilibrium. In addition, the void superlattices we obtained are not thermodynamically stable, and the free energy formulation in our model is isotropic (without any embedded spatial symmetry). Another analytical method is developed by the construction of a non-equilibrium potential to substitute the effects of source and sink terms $[13,18]$. Such a treatment works for specific cases (e.g., ballistic mixing). However, it may not be easily generalized to all kinds of sources and sinks. As a result, the above methods are not adapted in our current analysis, and a mathematical substitution of kinetic anisotropy by thermodynamic anisotropy is beyond the scope of this work.

\section{CONCLUSIONS}

The physical origin and formation mechanism of void superlattices under irradiation in metals and alloys are systematically investigated through phase field modeling and simula- 
tions. The major findings include:

- Depending on the irradiation conditions, there are three possible formation mechanisms of void superlattices, according to the kinetics for the characteristic length and that for symmetry development.

- The characteristic length of a void superlattice is determined by the interplay of thermodynamic driving forces and the production/reaction rates of voids and interstitials.

- The characteristic symmetry of a void superlattice is dictated by the 1-dimensional diffusion directions of self-interstitial atoms, which can be systematically analyzed and predicted in reciprocal space.

\section{ACKNOWLEDGEMENTS}

The authors gratefully acknowledge the support of U.S. Department of Energy, Office of Science, Basic Energy Sciences project - The Role of Anisotropy on the Self-Organization of Gas Bubble Superlattices. All calculations are performed using the Falcon supercomputing system at Idaho National Laboratory.

\section{APPENDIX A: DETERMINATION OF THE CRITICAL CONCENTRATION WAVE- LENGTH}

Starting from Eq. 15,

$$
R(k)=-M_{v} f^{\prime \prime} k^{2}-M_{v} \kappa k^{4}-Q
$$

when $R(k)>0$, the system will lose stability to the concentration wave with wave vector $k$, i.e. such a wave will develop. Assuming the first developed wave is $k_{c}, R(k)$ should reach maximum at $k_{c}$. The critical conditions in determining $k_{c}$ should include the following two equations,

$$
\begin{gathered}
\left.\frac{d R}{d k}\right|_{k=k_{c}}=0, \\
R\left(k_{c}\right)=0 .
\end{gathered}
$$


As a result, $f^{\prime \prime}=-2 \kappa k_{c}^{2}$ can be obtained from Eq. A2. Combined with Eq. A3, we can determine the critical wave vector,

$$
k_{c}=\left(\frac{Q}{\kappa M_{v}}\right)^{1 / 4}
$$

and the corresponding critical wave length is,

$$
\lambda_{c}=\frac{2 \pi}{k_{c}}=2 \pi\left(\frac{\kappa M_{v}}{Q}\right)^{1 / 4} .
$$

Even though $f^{\prime \prime}$ is not in Eq. A5 explicitly, it is the thermodynamic driving force for the spinodal decomposition, which should be large enough to overcome the barrier from recombination, i.e., $R(k) \geq 0$. With the expression of $k_{c}$ through Eq. A4 and expression of $R(k)$ through Eq. A1, the condition of $R(k) \geq 0$ leads to the following inequality,

$$
-f^{\prime \prime} \geq \sqrt{\frac{4 \kappa Q}{M_{v}}} .
$$

$Q=K c_{v}$ and $c_{v}=\sqrt{P / K}$ for steady state homogeneous vacancy concentration. Also $c_{v}$ should be inside the spinodal region, which leads to the inequality of Eq. 17.

\section{APPENDIX B: DETERMINATION OF VACANCY MOBILITY AND VOID IN- TERFACIAL ENERGY IN MO}

For Mo, the interfacial energy between void and matrix is reported as $\gamma=3.25 \mathrm{~J} / \mathrm{m}^{2}[61]$, and the heat of mixing is $E_{m i x}=3.03 \times 10^{10} \mathrm{~J} / \mathrm{m}^{3}$. Thus the gradient coefficient can be determined as [59]

$$
\kappa=\frac{9 \gamma^{2}}{32 \triangle f}=\frac{9 \gamma^{2}}{8 E_{m i x}}=3.92 \times 10^{-10} \mathrm{~J} / \mathrm{m}
$$

And the interface width can be determined as [41]

$$
w=2 \triangle c_{v} \sqrt{\frac{\kappa}{\triangle f}}=4.55 \times 10^{-10} \mathrm{~m} .
$$

According to the regular solution model, $\triangle f \sim 0.25 E_{m i x}$. The vacancy diffusivity in Mo can be described as [60],

$$
D_{v}=7.44 \times 10^{-8} \exp \frac{-1.45}{k_{b} T} m^{2} / s
$$


$k_{b}$ is the Boltzmann constant.

Vacancy mobility can be determined as follows, when a regular solution model is adopted in the free energy formulation.

$$
M_{v}=\frac{D_{v} V_{m}}{R T}
$$

where $R$ is the gas constant, and $V_{m}$ is the molar volume of Mo.

* Corresponding author: yongfeng.zhang@inl.gov

[1] D. Nagy, G. Konya, G. Szirmai, and P. Domokos. Dicke-model phase transition in the quantum motion of a bose-einstein condensate in an optical cavity. Phys. Rev. Lett., 104:130401, 2010.

[2] E. K. H. Salje, A. Graeme-Barber, M. A. Carpenter, and U. Bismayer. Lattice parameters, spontaneous strain and phase transitions in pb3(po4)2. Acta Cryst. B, 49:387-392, 1993.

[3] C. Petit, A. Taleb, and M. P. Pileni. Self-organization of magnetic nanosized cobalt particles. Adv. Mater., 10:259-261, 1998.

[4] Y. Gao, N. Zhou, D. Wang, and Y. Wang. Pattern formation during cubic to orthorhombic martensitic transformations in shape memory alloys. Acta Mater., 68:93-105, 2014.

[5] H. Fan, P. Werner, and M. Zacharias. Semiconductor nanowires: From self-organization to patterned growth. Small, 2:700-717, 2006.

[6] H. Liu, Y. Gao, Z. Xu, Y. M. Zhu, Y. Wang, and J.-F. Nie. Guided self-assembly of nanoprecipitates into mesocrystals. Sci. Rep., 5:16530, 2015.

[7] Y. Lu, C. Wang, Y. Gao, R. Shi, X. Liu, and Y. Wang. Microstructure map for self-organized phase separation during film deposition. Phys. Rev. Lett., 109:086101, 2012.

[8] Y. Gao, S. A. Dregia, and Y. Wang. A universal symmetry criterion for the design of high performance ferroic materials. Acta Mater., 127:438-449, 2017.

[9] G. Demange, M. Chamaillard, H. Zapolsky, Lavrskyi, A. Vaugeois, and L. Luneville. Generalization of the fourier-spectral eyre scheme for the phase-field equations: Application to self-assembly dynamics in materials. Comp. Mater. Sci., 144:11-22, 2018.

[10] A. Jaatinen and T. Ala-Nissila. Extended phase diagram of the three-dimensional phase field crystal model. J. Phys. Condens. Matter., 22:205402, 2010.

[11] M. Lavrskyi, H. Zapolsky, and A. G. Khachaturyan. Quasiparticle approach to diffusional 
atomic scale self-assembly of complex structures: from disorder to complex crystals and double-helix polymers. npj Comput. Mater, 2:15013, 2016.

[12] Y. M. Jin and A. G. Khachaturyan. Atomic density function theory and modeling of microstructure evolution at the atomic scale. J. Appl. Phys, 100:013519, 2006.

[13] G. Demange, H. Zapolsky, R. Patte, and M. Brunel. A phase field model for snow crystal growth in three dimensions. npj Comput. Mater., 3:15, 2017.

[14] L. Q. Chen and A. G. Khachaturyan. Dynamics of simultaneous ordering and phase separation and effect of long-range coulomb interactions. Phys. Rev. Lett., 70:1477-1480, 1993.

[15] A. M. Turing. The chemical basis of morphogenesis. Philos. Transact. Royal Soc. B, 237:37, 1952.

[16] M. C. Cross and P. C. Hohenberg. Pattern formation outside equilibrium. Rev. Mod. Phys., 65:851-1112, 1993.

[17] V. K. Vanag and I. R. Epstein. Stationary and oscillatory lcalized patterns, and subcritical bifurcations. Phys. Rev. Lett., 92:128301, 2004.

[18] R. A. Enrique and P. Bellon. Compositional patterning in systems driven by competing dynamics of different length scale. Phys. Rev. Lett., 84:2885, 2000.

[19] J. H. Evans. Observations of a regular void array in high purity molybdenum irradiated with 2 mev nitrogen ions. Nature, 29:403, 1971.

[20] I. Ipatova, P. T. Wady, S. M. Shubeita, C. Barcellini, A. Impagnatiello, and E. Jimenez-Melero. Radiation-induced void formation and ordering in ta-w alloys. J. Nucl. Mater., 495:343-350, 2017.

[21] J. Gan, B.D. Miller, D.D. Keiser Jr., A.B. Robinson, J.W. Madden, P.G. Medvedev, and D.M. Wachs. Microstructural characterization of irradiated u7mo/al5si dispersion fuel to high fission density. J. Nucl. Mater., 2014:434-445, 2010.

[22] K. Krishan. Void ordering in metals during irradiation. Philos. Mag. A, 45(3):401-417, 1982.

[23] N. M. Ghoniem, D. Walgraef, and S. J. Zinkle. Theory and experiment of nanostructure self-organization in irradiated materials. J. Comput. Aided Mater. Des., 8:1-38, 2001.

[24] J. W. Cahn. On spinodal decomposition. Acta Metall., 9:795, 1961.

[25] K. Malen and Bullough R. In Proc. Int. Conf. on Voids Formed by Irradiation of Reactor Materials, Reading, 1971.

[26] M. J. Imada. Void lattice formation - spinodal decomposition of vacancies. Phys. Soc. Jap., 
45:1443, 1978.

[27] M. S. Veshchunov and L. V. Matveev. Spinodal decomposition of a solid solution under irradiation and void ordering. Atomic Energy, 76:29, 1994.

[28] H. C. Yu and W. Lu. Dynamics of the self-assembly of nanovoids and nanobubbles in solids. Acta Mater., 53(6):1799-1807, 2005.

[29] K. Krishan. Kinetics of void-lattice formation in metals. Nature, 287(5781):420-421, 1980.

[30] G. Martin. Instability of crystalline solids under irradiation. Philos. Mag., 32:615, 1975.

[31] A. V. Barashev and S. I. Golubov. On the onset of void ordering in metals under neutron or heavy-ion irradiation. Philos. Mag., 90:1787-1797, 2010.

[32] D. Walgraef, J. Lauzeral, and N. M. Ghoniem. Theory and numerical simulations of defect ordering in irradiated materials. Phys. Rev. B, 53(22):14782-14794, 1996.

[33] Y. Gao, Y. Zhang, D. Schwen, C. Jiang, C. Sun, and J. Gan. Self-organization of void superlattices: atomic scale perspective and theoretical prediction. Under review, 2017.

[34] A. J. E. Forreman. Report aere-r-7135. Technical report, Atomic Energy Research Establishment, 1972 .

[35] C. H. Woo and W. Frank. A theory of void-lattice formation. J. Nucl. Mater., 137:7-21, 1985.

[36] J. H. Evans. Void and bubble lattice formation in molybdenum - a mechanism based on two-dimensional self-interstitial diffusion. J. Nucl. Mater., 119(2-3):180-188, 1983.

[37] H. L. Heinisch and B. N. Singh. Kinetic Monte Carlo simulations of void lattice formation during irradiation. Philos. Mag., 83(31-34):3661-3676, 2003.

[38] S. Y. Hu and C. H. Henager. Phase-field modeling of void lattice formation under irradiation. J. Nucl. Mater., 394(2-3):155-159, 2009.

[39] S. Y. Hu, D. Burkes, C. A. Lavender, D. J. Senor, and Z. Xu. Formation mechanism of gas bubble superlattice in umo metal fuels: Phase-field modelling investigation. J. Nucl. Mater., 479:202-215, 2016.

[40] V. I. Dubinko, A. V. Tur, A. A. Turkin, and V. V. Yanovskij. A mechanism of formation and properties of the void lattice in metals under irradiation. J. Nucl. Mater., 161(1):57-71, 1989.

[41] J. W. Cahn and J. E Hillard. Free energy of a nonuniform system. i. interfacial free energy. J. Chem. Phys., 28:258, 1958.

[42] R. Bullough, B. L. Eyre, and K. Krishan. Cascade damage effects on swelling of irradiated materials. Proc. Royal Soc. Lond. A, 346(1644):81-102, 1975. 
[43] S. Rokkam, A. El-Azab, P. Millett, and D. Wolf. Phase field modeling of void nucleation and growth in irradiated metals. Modell. Simul. Mater. Sci. Eng., 17:064002, 2009.

[44] P. C. Millett, S. Rokkam, A. El-Azab, M. Tonks, and D. Wolf. Void nucleation and growth in irradiated polycrystalline metals: a phase-field model. Modell. Simul. Mater. Sci. Eng., 17:064003, 2009 .

[45] S. Y. Hu and C. H. Henager. Phase-field simulation of void migration in a temperature gradient. Acta Mater., 58(9):3230-3237, 2010.

[46] Y. Li, S. Hu, X. Sun, F. Gao, C. H. Henager Jr., and M. Khaleel. Phase-field modeling of void migration and growth kinetics in materials under irradiation and temperature field. $J$. Nucl. Mater., 407:119-125, 2010.

[47] A. A. Semenov and C. H. Woo. Interfacial energy in phase-field emulation of void nucleation and growth. J. Nucl. Mater., 411:144-149, 2011.

[48] P. C. Millett, A. El-Azab, S. Rokkam, M. Tonks, and D. Wolf. Phase-field simulation of irradiated metals part i: Void kinetics. Comput. Mater. Sci., 50:949-959, 2011.

[49] A. A. Semenov and C. H. Woo. Phase-field modeling of void formation and growth under irradiation. Acta Mater., 60:6112-6119, 2012.

[50] Z. H. Xiao, A. A. Semenov, C. H. Woo, and S. Q. Shi. Single void dynamics in phase field modeling. J. Nucl. Mater., 439:25-32, 2013.

[51] A. El-Azab, K. Ahmed, S. Rokkam, and T. Hochrainer. Diffuse interface modeling of void growth in irradiated materials. mathematical, thermodynamic and atomistic perspectives. Curr. Opin. Solid State Mater. Sci., 18:90-98, 2014.

[52] N. Wang, S. Rokkam, T. Hochrainer, M. Pernice, and A. El-Azab. Asymptotic and uncertainty analyses of a phase field model for void formation under irradiation. Comput. Mater. Sci., $89: 165-175,2014$.

[53] A. A. Semenov and C. H. Woo. Modeling void development in irradiated metals in the phasefield framework. J. Nucl. Mater., 454:60-68, 2014.

[54] Y. Li, S. Hu, X. Sun, and M. Stan. A review: applications of the phase field method in predicting microstructure and property evolution of irradiated nuclear materials. NPJ Comput. Mater., 3:16, 2017.

[55] D. Schwen, L. K. Aagesen, J. W. Peterson, and M. R. Tonks. Rapid multiphase-field model development using a modular free energy based approach with automatic differentiation in 
moose/marmot. Comput. Mater. Sci., 132:36-45, 2017.

[56] D. R. Gaston, C. J. Permann, J. W. Peterson, A. E. Slaughter, D. Andrs, Y. Wang, M. P. Short, D. M. Perez, M. R. Tonks, J. Ortensi, and R. C. Martineau. Physics-based multiscale coupling for full core nuclear reactor simulation. Ann. Nucl. Energy, 84:45-54, 2015.

[57] D. A. Knoll and D. E. Keyes. Jacobian-free newton-krylov methods: a survey of approaches and applications. J. Comput. Phys, 193:357-397, 2004.

[58] B.S. Kirk, J.W. Peterson, R.H. Stogner, and G.F. Carey. libmesh: a c++ library for parallel adaptive mesh refinement/coarsening simulations. Eng. Comput., 22:237-254, 2006.

[59] L. Q. Cheng. Phase-fieldmodels formicrostructure evolution. Annu. Rev. Mater. Res., 32:113$140,2002$.

[60] D. Nguyen-Manh, A. P. Horsfield, and S. L. Dudarev. Self-interstitial atom defects in bcc transition metals: Group-specific trends. Phys. Rev. B, 73:020101, 2006.

[61] L. Vitos, A. V. Ruban, H. L. Skriver, and J. Kollar. The surface energy of metals. Surface Science, 411:186-202, 1998.

[62] W. Pfeiler (Eds.). Alloy physics: A comprehensive reference. Wiley-VCH Verlag GmbH \& Co. KGaA, 2007.

[63] Yongfeng Zhang, Hanchen Huang, Paul C. Millett, Michael Tonks, Dieter Wolf, and Simon R. Phillpot. Atomistic study of grain boundary sink strength under prolonged electron irradiation. J. Nucl. Mater., 422:69-76, 2012.

[64] J. Moteff, V.K. Sikka, and H. Jang. The physics of irradiation produced voids. In Consultant Symp. on the physics of irradiation produced voids, 1975.

[65] B. A. Loomis, S. B. Gerber, and A. Taylor. Void ordering in ion-irradiated nb and nb-1\%zr. J. Nucl. Mater., 68:19-31, 1977.

[66] S. J. Zinkle and B. N. Singh. Microstructure of cuni alloys neutron irradiated at 210c and 420c to 14 dpa. J. Nucl. Mater., 283:306-312, 2000.

[67] J. Gan, B. D. Miller, D. D. Keiser Jr., A. B. Robinson, J. W. Madden, P. G. Medvedev, and D. M. Wachs. Microstructural characterization of irradiated $\mathrm{u}-7 \mathrm{mo} / \mathrm{al}-5 \mathrm{si}$ dispersion fuel to high fission density. J. Nucl. Mater., 454:434-445, 2014.

[68] R. W. Harrison, G. Greaves, J. A. Hinks, and S. E. Donnelly. Engineering self-organising helium bubble lattices in tungsten. Sci. Rep., 7:7724, 2017.

[69] A. G. Khachaturyan. Theory of structural transformations in solids. Wiley, New York, 1983. 
[70] N. Provatas and K. Elder. Phase-Field Methods in Materials Science and Engineering. WileyVCH, Singapore, 2010. 


\section{FIGURE LEGEND}

FIG. 1. Simulation results of the formation of a square void superlattice. Dimensionless time $t^{*}$ : (a) 836, (b) 876, (c) 948, (d) 1167, (e) 1351, (f) 2000.

FIG. 2. Plots of SIA concentration fields during the void superlattice formation shown in FIG.1. $c_{1}$ and $c_{2}$ at $t^{*}=836$ (a and d), 1167 (b and e), 2000 (c and f).

FIG. 3. Simulation results of the formation of a square void superlattice through coarsening and coalescence. Dimensionless time $t^{*}$ : (a) 640, (b) 897, (c) 1331, (d) 2060, (e) 2474, (f) 4096 .

FIG. 4. Simulation results of the formation of a void superlattice through nucleation and growth. Dimensionless time $t^{*}$ : (a) 365, (b) 640, (c) 872, (d) 986, (e) 1125, (f) 2631.

FIG. 5. Simulation results of the formation of a hexagonal void superlattice through coarsening and coalescence. Dimensionless time $t^{*}$ : (a) 702, (b) 919, (c) 1167, (d) 2000, (e) 4936, (f) 7474 .

FIG. 6. Simulation results of void superlattices in 3D. (a) a BCC void superlattice, (b) an FCC void superlattice.

FIG. 6. Simulation results of the formation of void superlattices with different lattice parameters.

FIG. 7. Simulation results of the formation of a void superlattice with considerable coarsening and coalescence (Fourier transformation patterns are inset). Dimensionless time $t^{*}$ : (a) 66, (b) 87, (c) 171, (d) 229, (e) 326, (f) 2000.

FIG. 8. Simulation results of the formation of a void superlattice through nucleation and growth (Fourier transform patterns are inset). Dimensionless time $t^{*}$ : (a) 1553, (b) 2581, (c) 3112, (d) 3690, (e) 4016, (f) 5886.

FIG. 9. Plots of SIA concentration distributions during the formation of a void superlattice through nucleation and growth. $c_{1}$ and $c_{2}$ at $t^{*}=1553$ (a and d), 3112 (b and e), 5886 (c and $\mathrm{f})$.

FIG. 10. A $P-K$ diagram showing the selection of void superlattice formation mechanism. The colored dots represent the simulation conditions under which a certain formation mechanism is identified, i.e., red: Mechanism (I), blue: Mechanism (II), green: Mechanism (III). $M_{v}^{*}=0.3$ for the simulations.

FIG. 11. Simulation results of void superlattices with different symmetry and Fourier 
transform patterns (inset). (a) a square void superlattice, (b) a hexagonal void superlattice. 
(a)

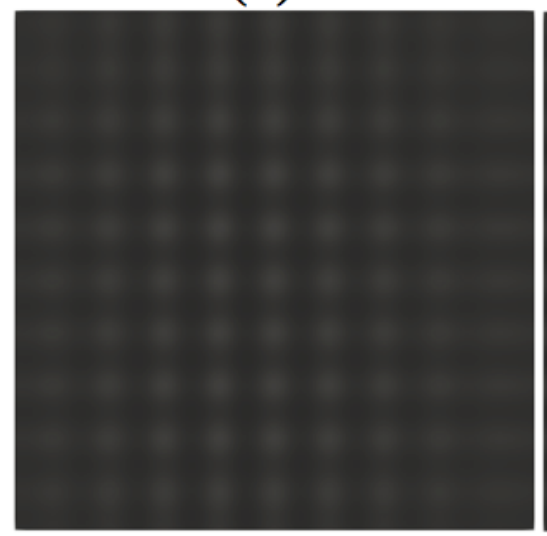

(d)

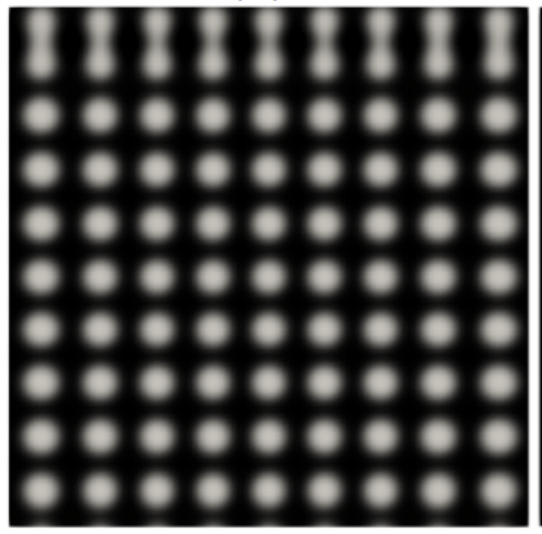

(b)

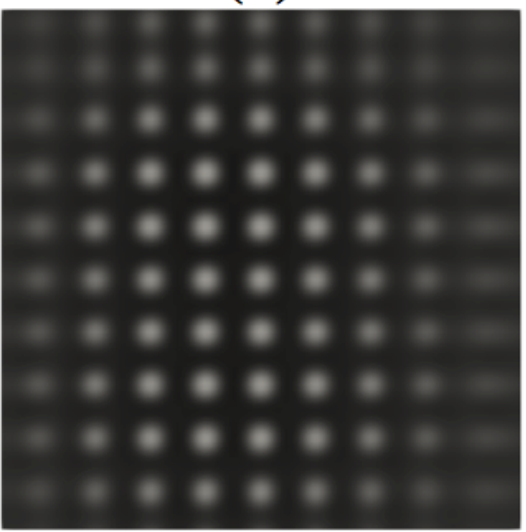

(e)

(c)

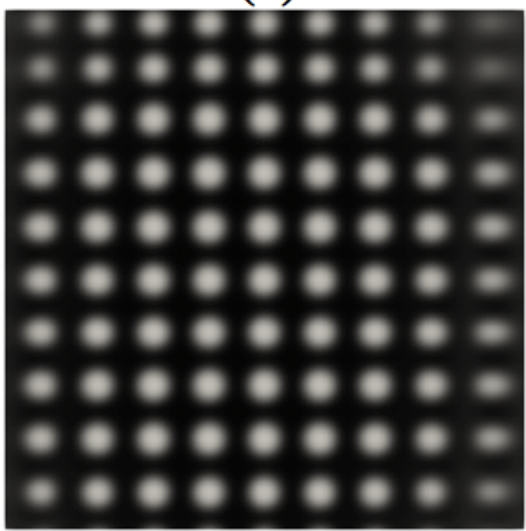

(f)

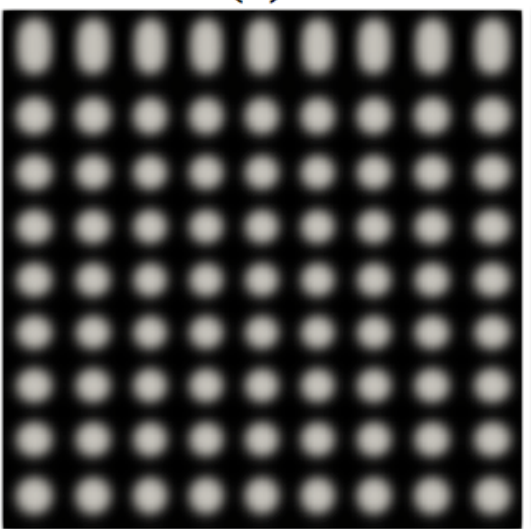

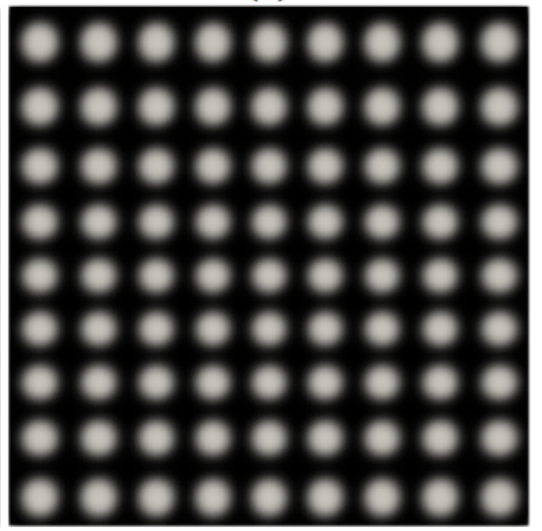

FIG. 1: Simulation results of the formation of a square void superlattice. Dimensionless time $t^{*}$ : (a) 836, (b) 876, (c) 948, (d) 1167, (e) 1351, (f) 2000. 
(a)

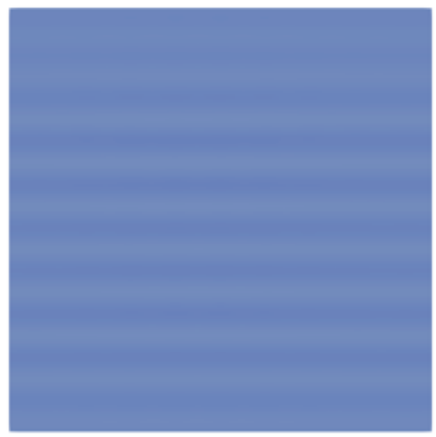

(d)

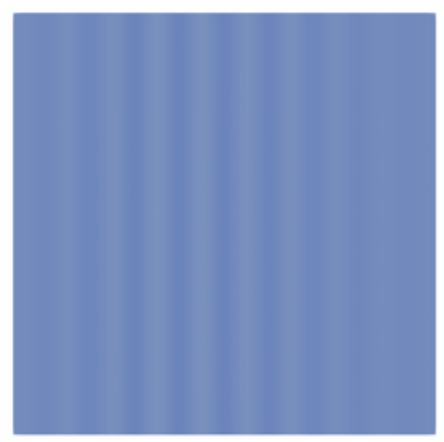

(b)

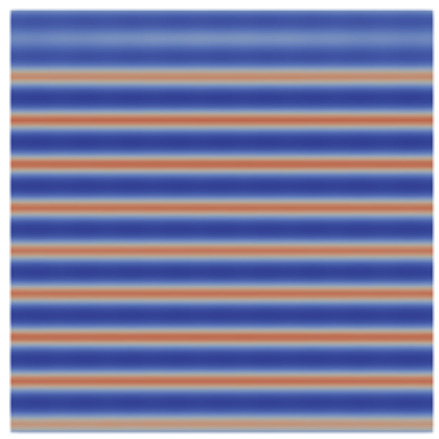

(e) (c)

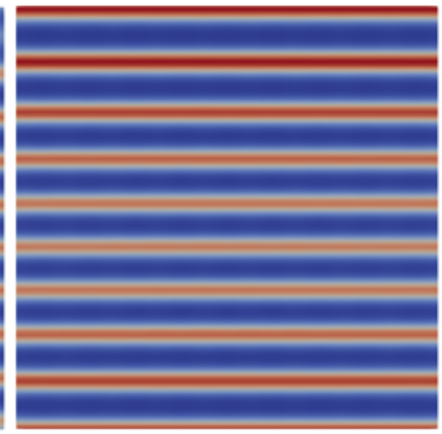

(f)

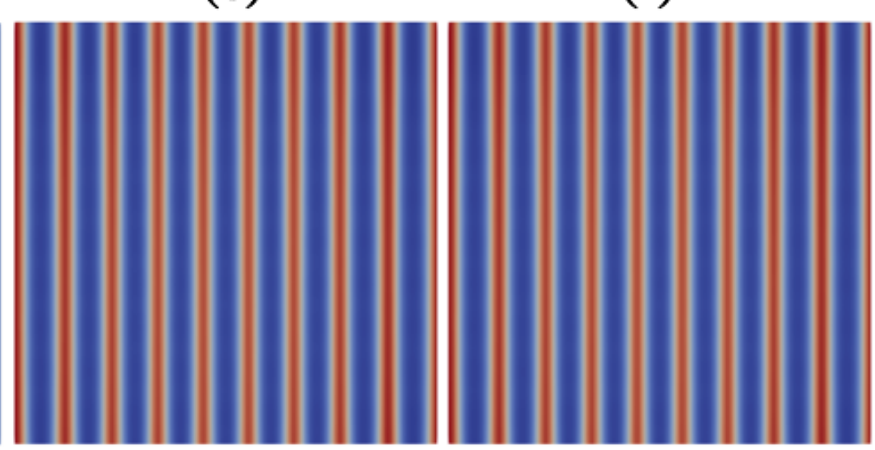

$\mathbf{c}_{1}$
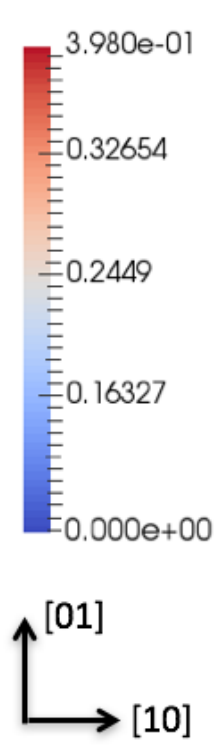

FIG. 2: Plots of SIA concentration fields during the void superlattice formation shown in FIG. 1. $c_{1}$ and $c_{2}$ at $t^{*}=836$ (a and d), 1167 ( $\mathrm{b}$ and e), 2000 (c and $\mathrm{f}$ ). 
(a)

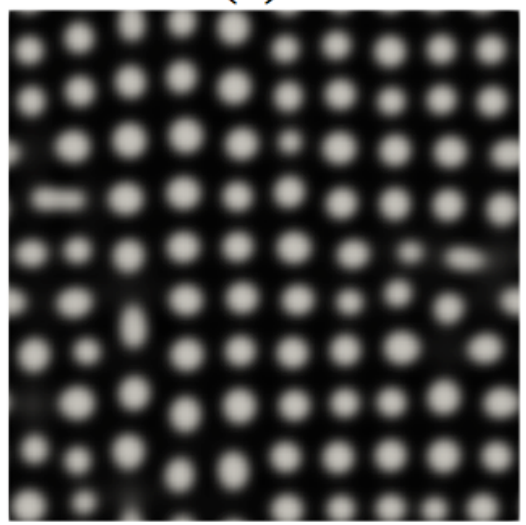

(d)

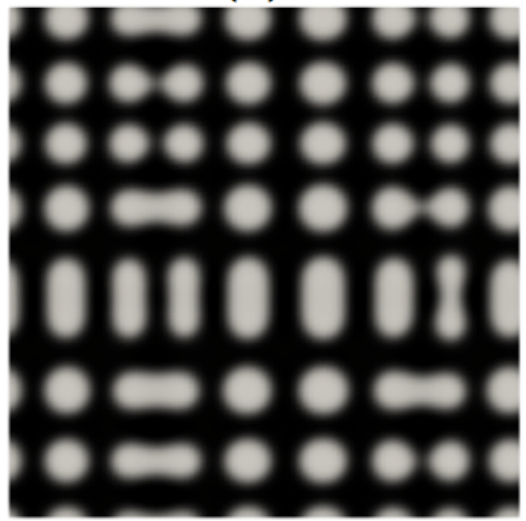

(b)

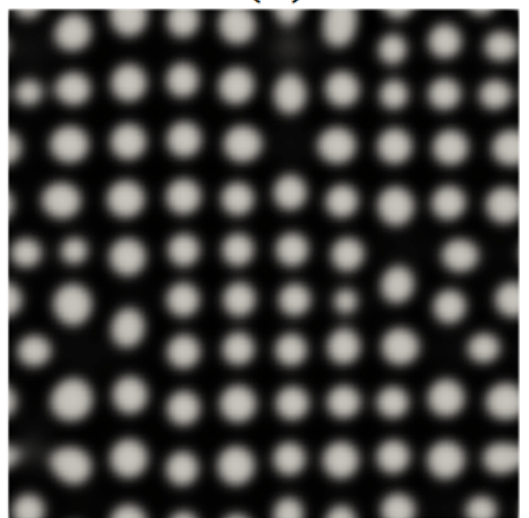

(e)

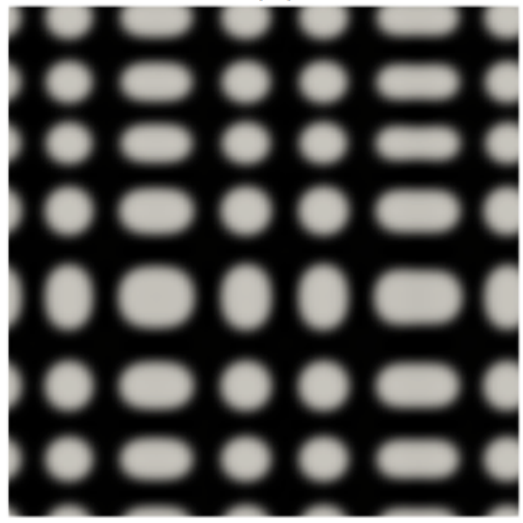

(c)

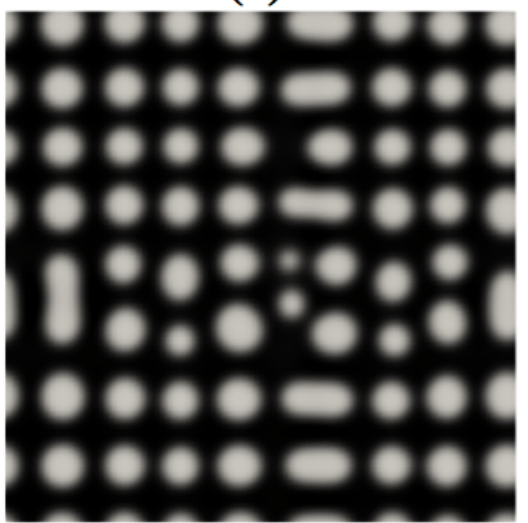

(f)

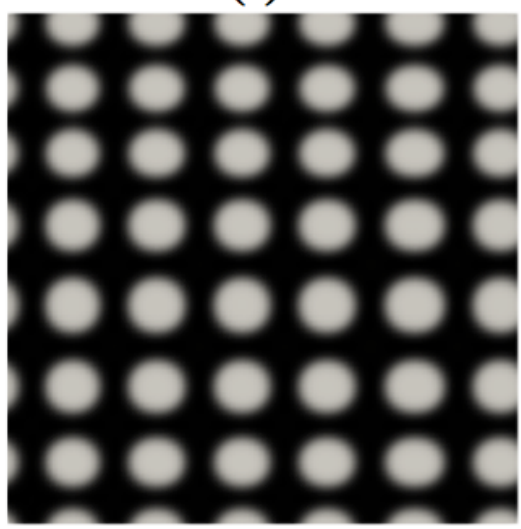

FIG. 3: Simulation results of the formation of a square void superlattice through coarsening and coalescence. Dimensionless time $t^{*}$ : (a) 640, (b) 897, (c) 1331, (d) 2060, (e) 2474, (f) 4096. 
(a)

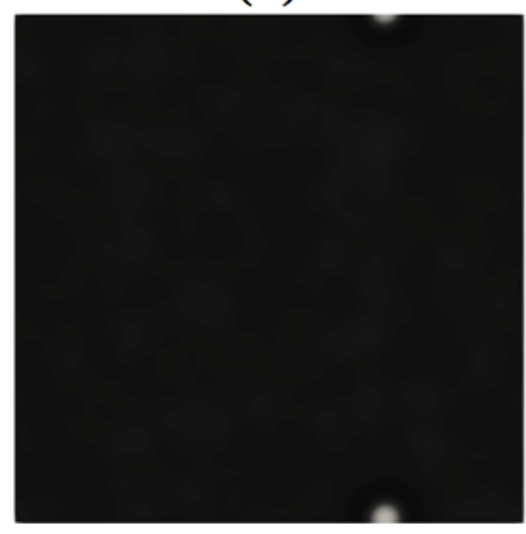

(d)

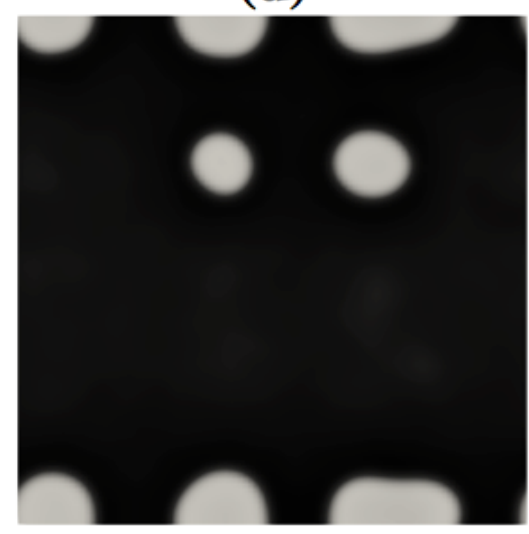

(b)

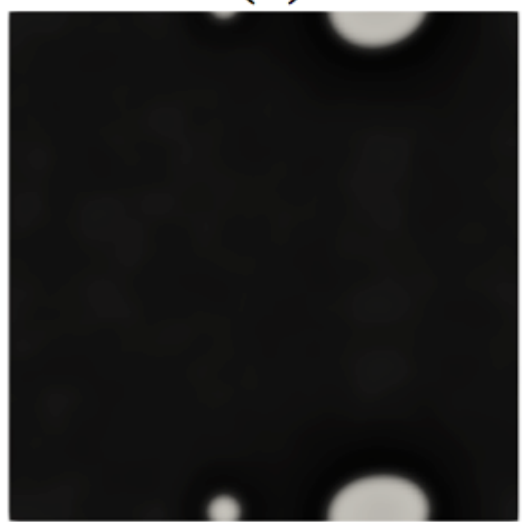

(e)

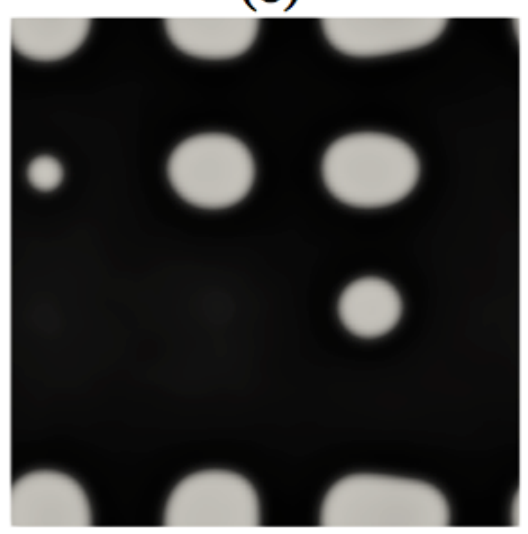

(c)

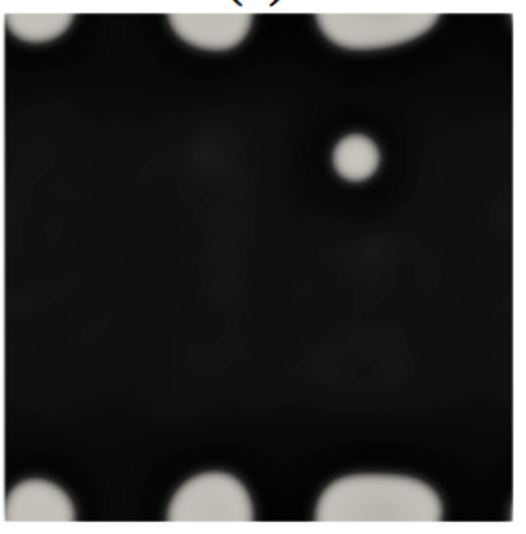

(f)

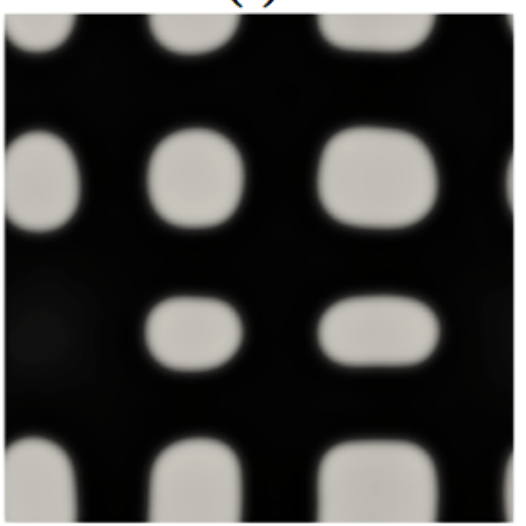

FIG. 4: Simulation results of the formation of a void superlattice through nucleation and growth. Dimensionless time $t^{*}$ : (a) 365, (b) 640, (c) 872, (d) 986, (e) 1125, (f) 2631. 
(a)

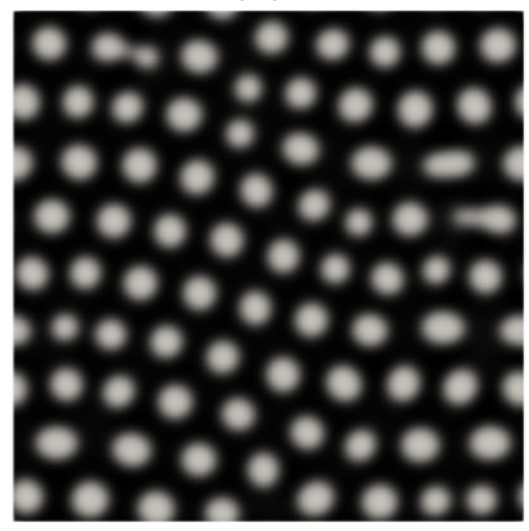

(d)

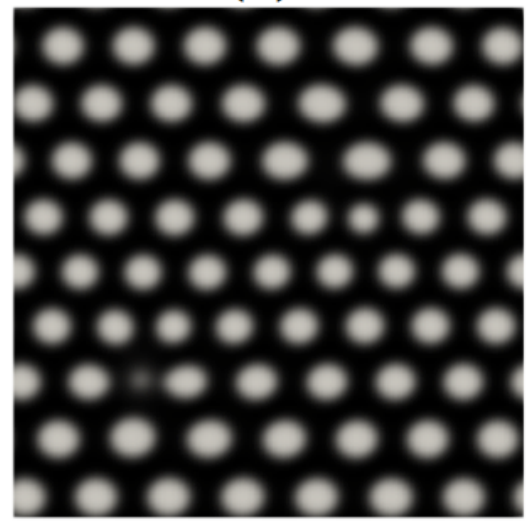

(b)

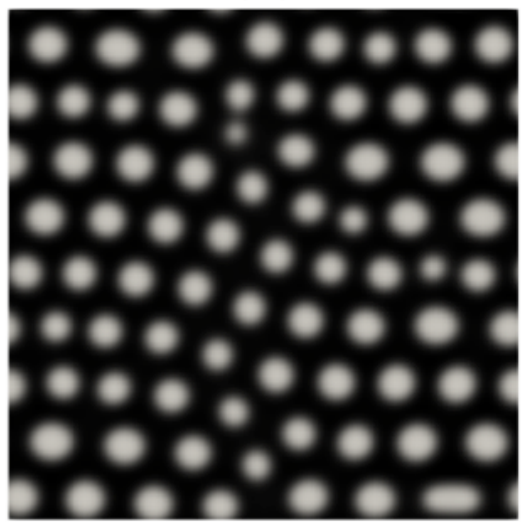

(e)

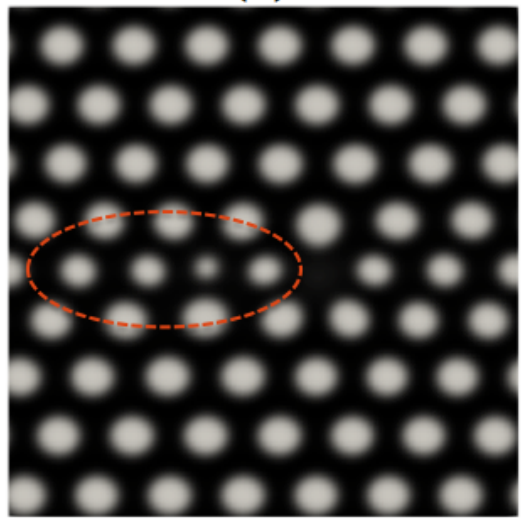

(c)

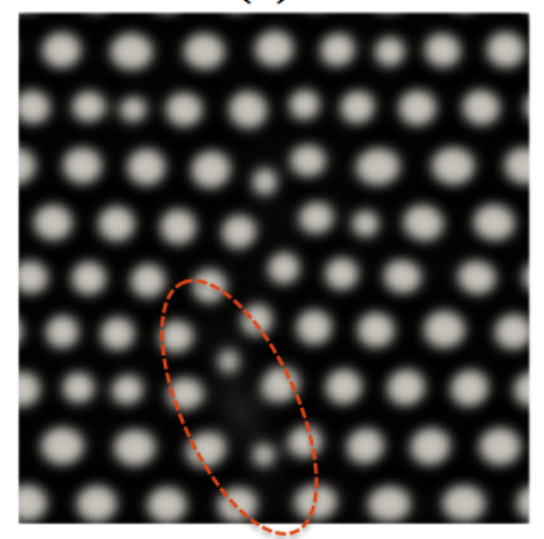

(f)

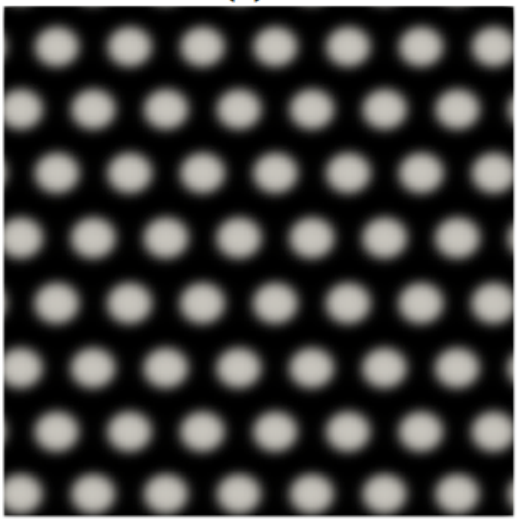

FIG. 5: Simulation results of the formation of a hexagonal void superlattice through coarsening and coalescence. Dimensionless time $t^{*}$ : (a) 702, (b) 919, (c) 1167, (d) 2000, (e) 4936, (f) 7474 .

(a)

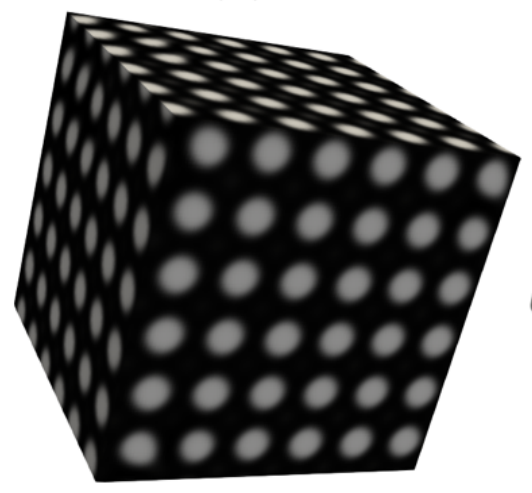

(b)

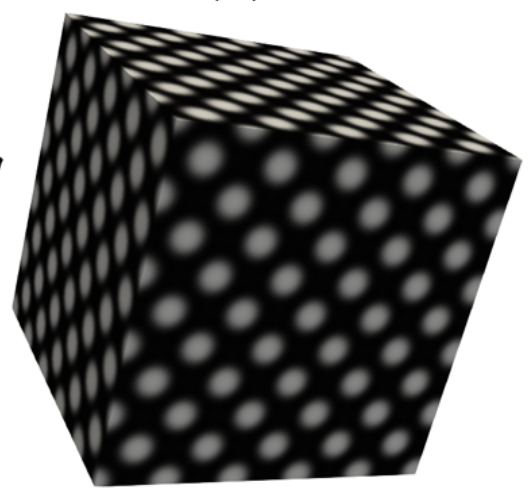

fig10

FIG. 6: Simulation results of void superlattices in 3D. (a) a BCC void superlattice, (b) an FCC void superlattice. 
(a)

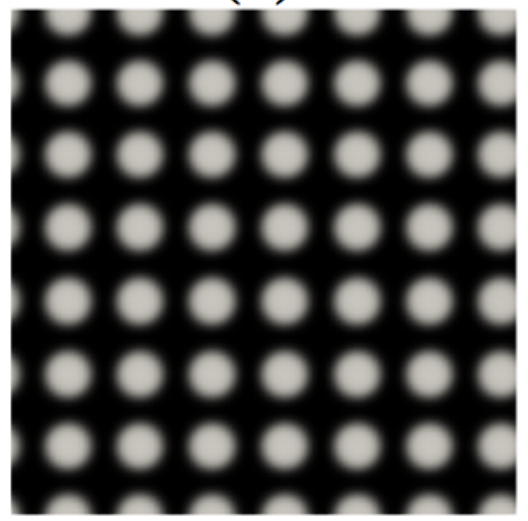

(c)

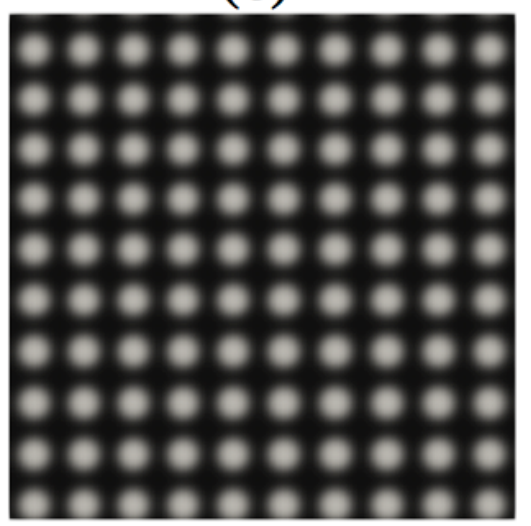

(b)

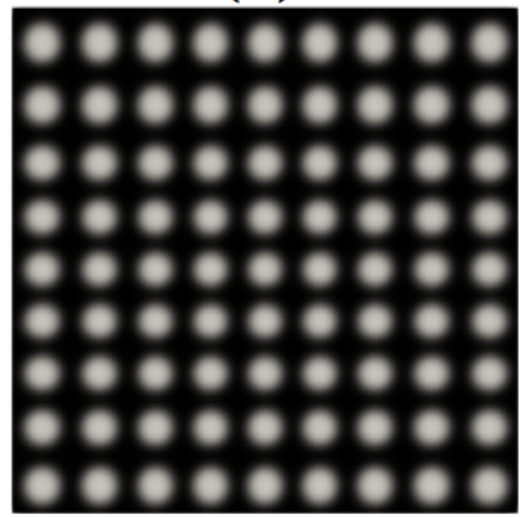

(d)

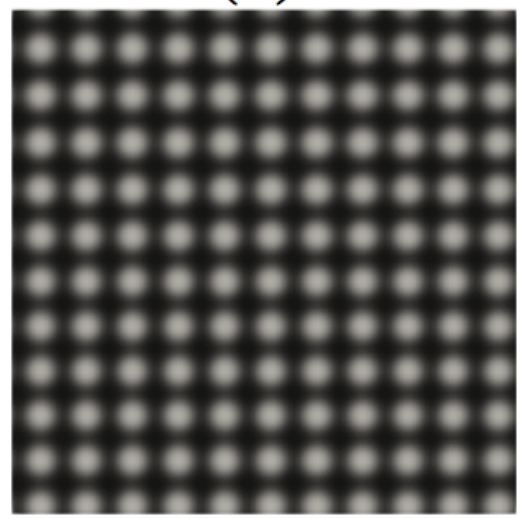

FIG. 7: Simulation results of the formation of void superlattices with different lattice parameters. 
(a)

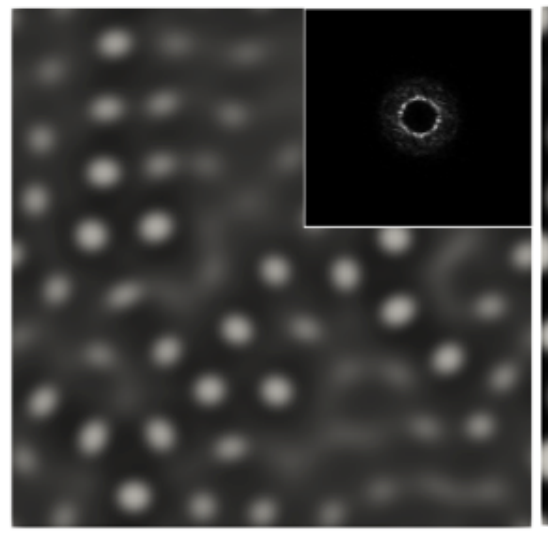

(d)

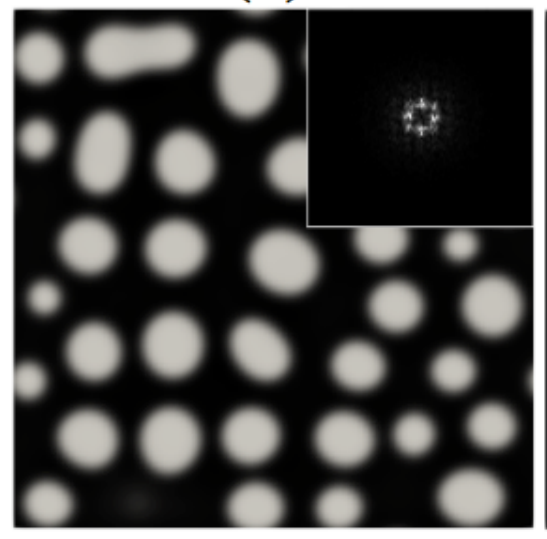

(b)

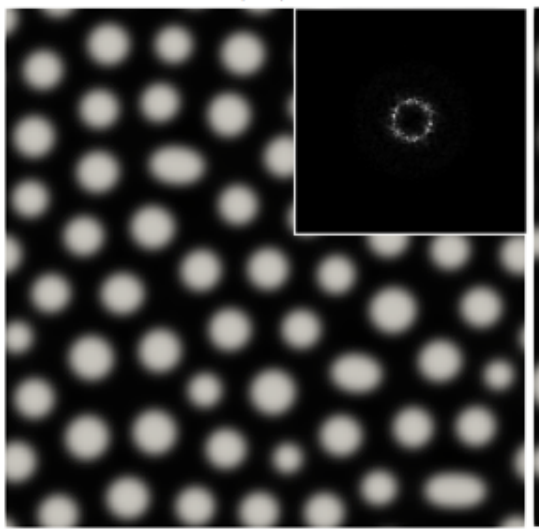

(e)

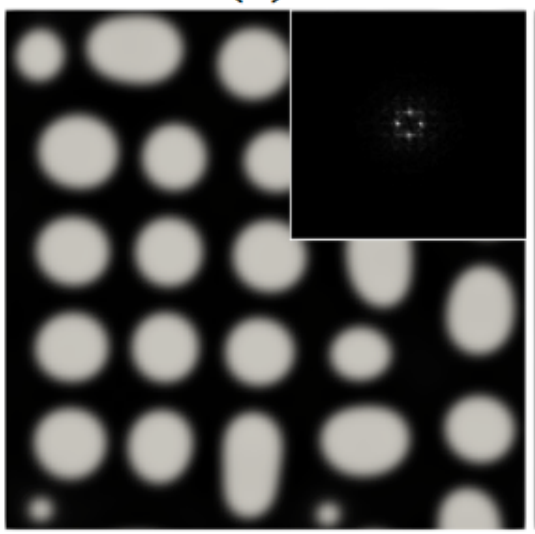

(c)

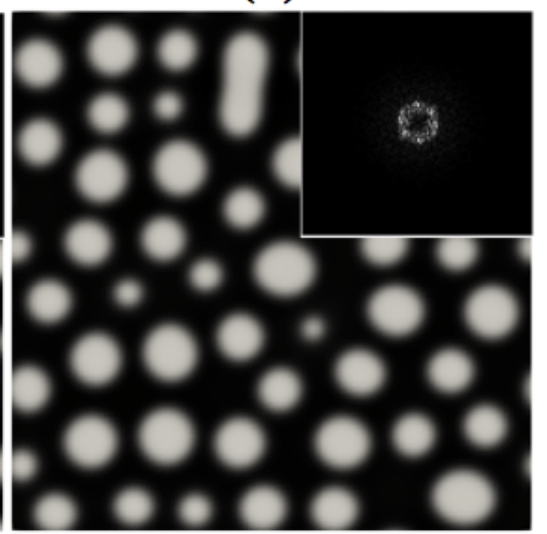

(f)

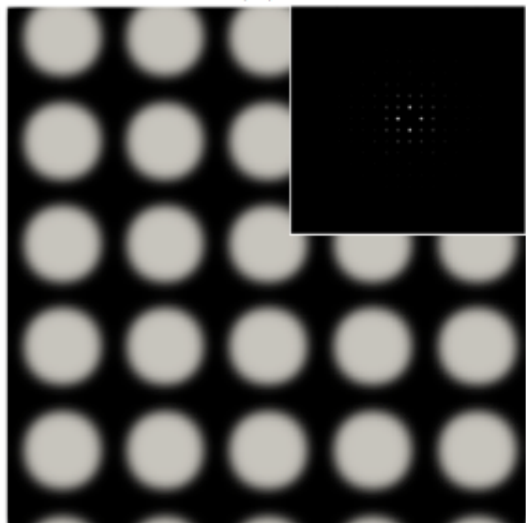

FIG. 8: Simulation results of the formation of a void superlattice with considerable coarsening and coalescence (Fourier transformation patterns are inset). Dimensionless time

$$
t^{*} \text { : (a) 66, (b) 87, (c) 171, (d) 229, (e) 326, (f) } 2000 .
$$


(a)

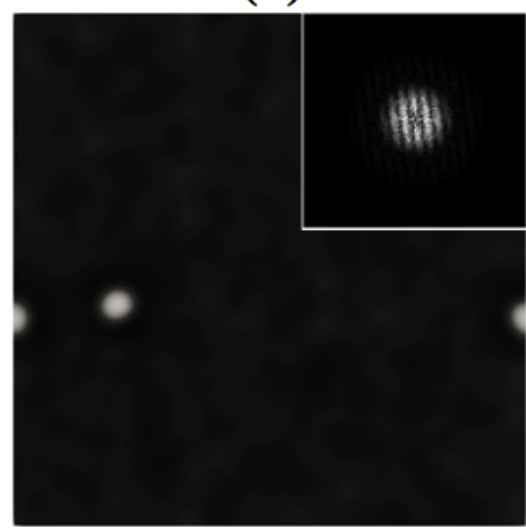

(d)

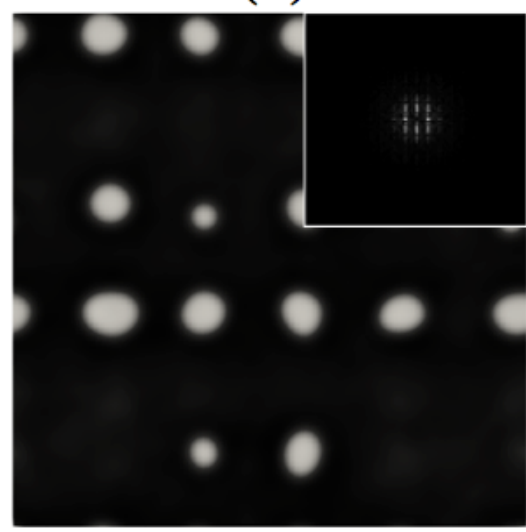

(b)

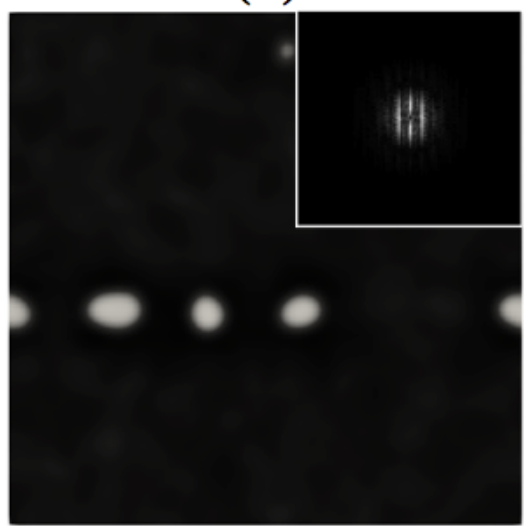

(e)

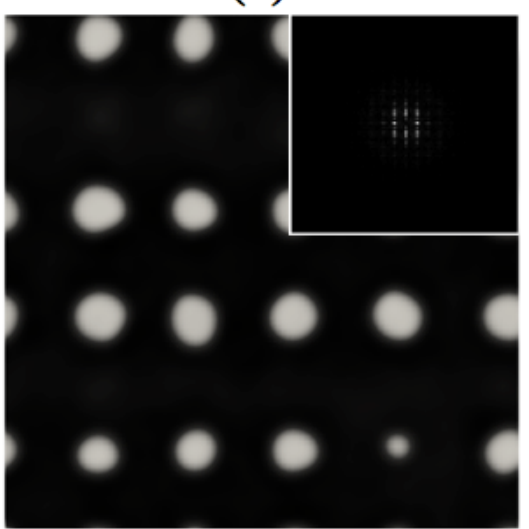

(c)

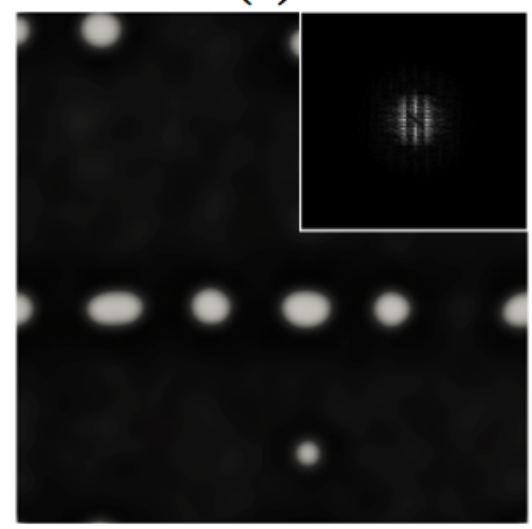

(f)

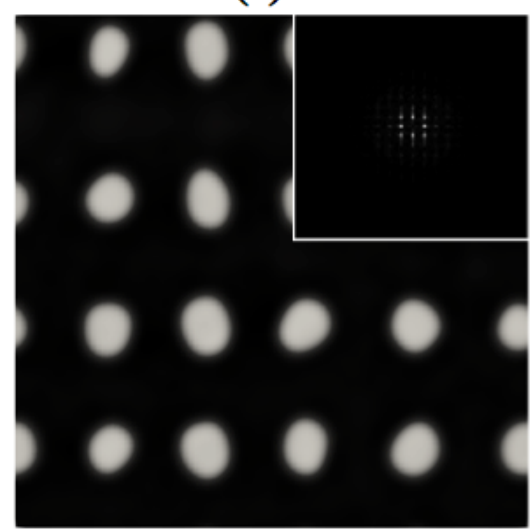

FIG. 9: Simulation results of the formation of a void superlattice through nucleation and growth (Fourier transform patterns are inset). Dimensionless time $t^{*}$ : (a) 1553, (b) 2581, (c) 3112, (d) 3690, (e) 4016, (f) 5886. 
(a)

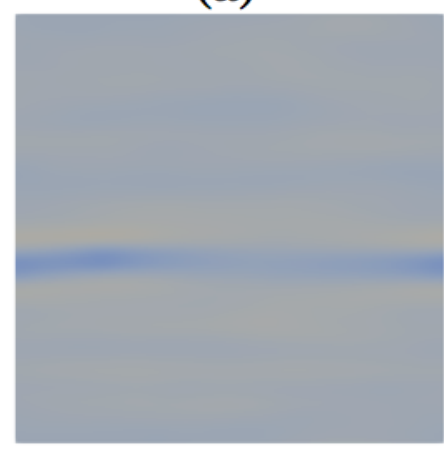

(d)

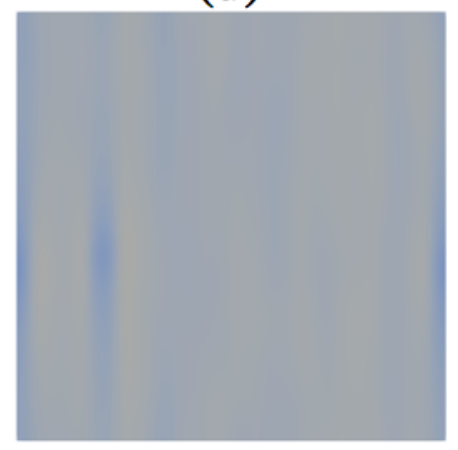

(b)

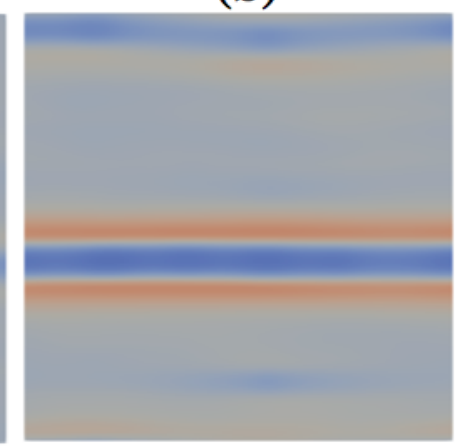

(e)

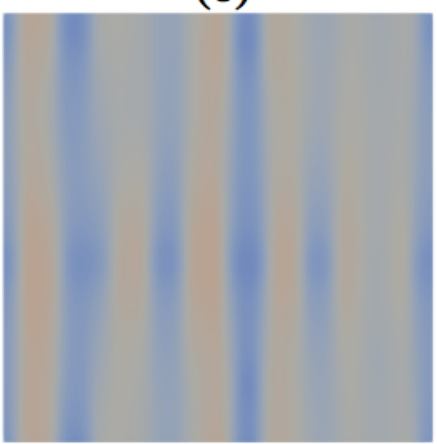

(c)

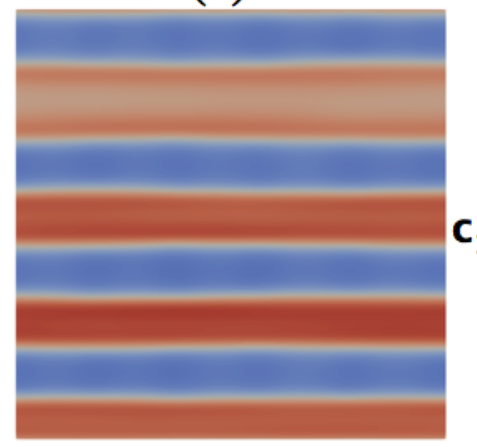

(f)

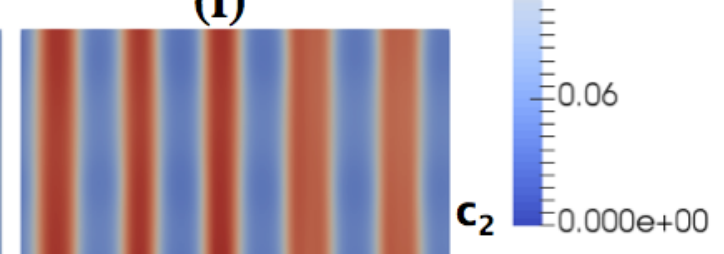

FIG. 10: Plots of SIA concentration distributions during the formation of a void superlattice through nucleation and growth. $c_{1}$ and $c_{2}$ at $t^{*}=1553$ (a and d), 3112 (b and e), 5886 (c and f).

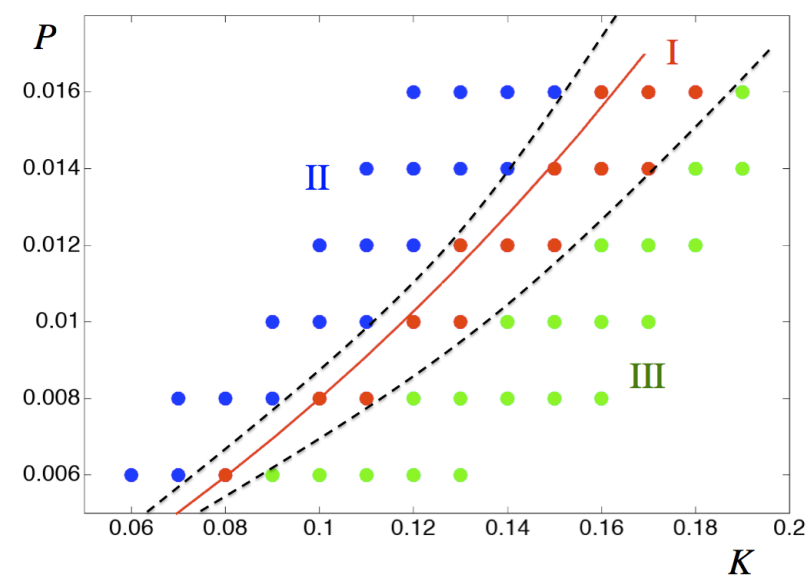

FIG. 11: A $P-K$ diagram showing the selection of void superlattice formation mechanism. The colored dots represent the simulation conditions under which a certain formation mechanism is identified, i.e., red: Mechanism (I), blue: Mechanism (II), green: Mechanism (III). $M_{v}^{*}=0.3$ for the simulations. 
(a)

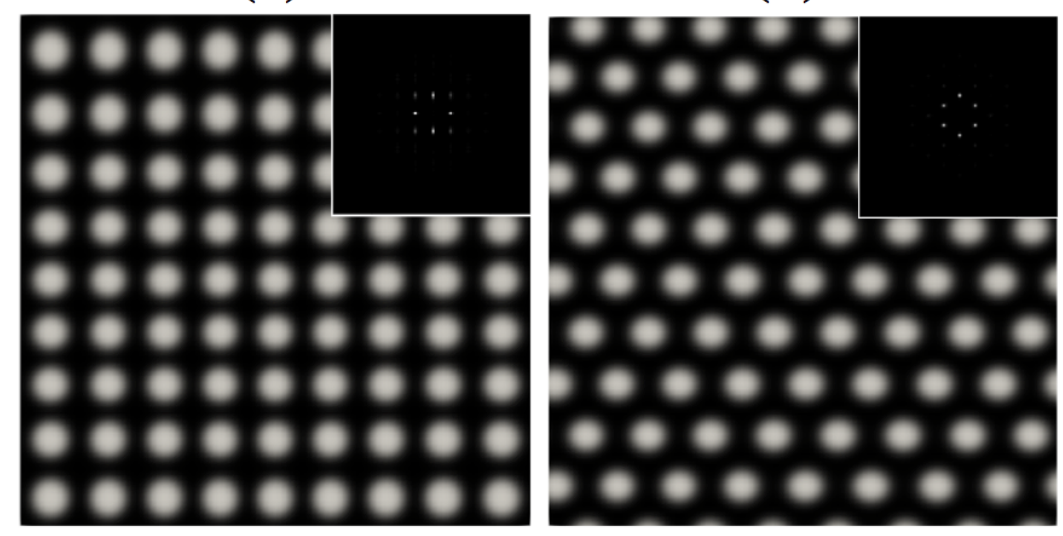

FIG. 12: Simulation results of void superlattices with different symmetry and Fourier transform patterns (inset). (a) a square void superlattice, (b) a hexagonal void superlattice. 
TABLE I: Dimensionless parameters used in phase field modeling and simulations

\begin{tabular}{llllll}
\hline FIG\# & $P^{*}$ & $K^{*}$ & $M_{v}^{*}$ & $\kappa^{*}$ & D-symmetry \\
\hline FIG.1 & 0.016 & 0.16 & 0.3 & 0.5 & 4-fold \\
\hline FIG.2 & 0.016 & 0.16 & 0.3 & 0.5 & 4-fold \\
\hline FIG.3 & 0.006 & 0.06 & 0.3 & 0.5 & 4-fold \\
\hline FIG.4 & 0.007 & 0.16 & 1.0 & 0.5 & 4-fold \\
\hline FIG.5 & 0.008 & 0.08 & 0.3 & 0.5 & 6-fold \\
\hline FIG.6(a) & 0.016 & 0.16 & 0.3 & 0.5 & BCC \\
\hline FIG.6(b) & 0.016 & 0.16 & 0.3 & 0.5 & FCC \\
\hline FIG.7(a) & 0.016 & 0.22 & 0.9 & 0.5 & 4-fold \\
\hline FIG.7(b) & 0.016 & 0.16 & 0.3 & 0.5 & 4-fold \\
\hline FIG.7(c) & 0.016 & 0.13 & 0.17 & 0.5 & 4-fold \\
\hline FIG.7(d) & 0.016 & 0.10 & 0.11 & 0.5 & 4-fold \\
\hline FIG.8 & 0.016 & 0.16 & 3 & 0.5 & 4-fold \\
\hline FIG.9 & 0.007 & 0.16 & 0.3 & 0.5 & 4-fold \\
\hline FIG.10 & 0.007 & 0.16 & 0.3 & 0.5 & 4-fold \\
\hline FIG.12(a) & 0.016 & 0.16 & 0.3 & 0.5 & 4-fold \\
\hline FIG.12(b) & 0.016 & 0.16 & 0.3 & 0.5 & 6-fold \\
\hline
\end{tabular}

TABLE II: Lattice parameters of void superlattice predicted in phase field simulations and analytical theory

\begin{tabular}{lll}
\hline Example & Simulation (FIG. 7) & Theory (Eq. 16) \\
\hline FIG.7(a) & 11.44 & 10.41 \\
\hline FIG.7(b) & 8.89 & 8.26 \\
\hline FIG.7(c) & 8.00 & 7.32 \\
\hline FIG.7(d) & 7.27 & 6.80 \\
\hline
\end{tabular}

